\title{
A survey on CAD methods in 3D garment design
}

\author{
Yong-Jin Liu ${ }^{\mathrm{a}, *}$, Dong-Liang Zhang ${ }^{\mathrm{b}}$, Matthew Ming-Fai Yuen ${ }^{\mathrm{c}}$ \\ a Department of Computer Science and Technology, Tsinghua National Lab for Information Science and Technology, Tsinghua University, Beijing, China \\ ${ }^{\mathrm{b}}$ Livesforce Co. Ltd, Hangzhou, China \\ ${ }^{\mathrm{c}}$ Department of Mechanical Engineering, The Hong Kong University of Science and Technology, Hong Kong, China
}

\section{A R T I C L E I N F O}

\section{Keywords:}

Garments

CAD methods

Feature modeling

\begin{abstract}
A B S T R A C T
With the advance in virtual reality applications, garment industry has strived for new developments. This paper reviews state-of-the-art CAD methods in 3D garment design. A large range of techniques are selected and organized into several key modules which form the core of a 3D garment design technology platform. In each module, basic techniques are presented first. Then advanced developments are systematically discussed and commented. The selected key modules - digital human modeling, 3D garment design and modification, numerical integration of draping, 2D pattern generation, geometric details modeling, parallel computation and GPU acceleration - are discussed in turn. Major challenges and solutions that have been addressed over the years are discussed. Finally, some of the ensuing challenges in 3D garment CAD technologies are outlined.
\end{abstract}

(c) 2010 Elsevier B.V. All rights reserved.

\section{Introduction}

Garment CAD technology is the use of computer technology to assist the design of garment product. Compared to other mechanical product, garment CAD has to address some special issues. Firstly, it models soft material with low bending stiffness rather than rigid solid objects. Secondly, garment components such as collar and sleeve, are assembled together following specific pattern-making rules which are totally different from the conventional assembly methods. Thirdly, while the garment is constructed from 2D patterns, the quality of fit is evaluated on 3D human models. Based on these observations, garment CAD is a unique research area that has attracted considerable attentions.

Early garment CAD focused on 2D pattern drafting and modification. The pattern-making techniques in 2D CAD systems mainly consist of two parts [117]: (1) individual pattern generation based on parametric design; (2) individual pattern altering based on grading rules. Since garment is more flexible than other industrial products, the constraint types used in both parametric design and the grading rules depend on pattern makers' experience which is case-sensitive: for example, different experiences are needed for different apparel patterns such as suit, jean and shirt. The well recognized flowchart of $2 \mathrm{D}$ garment $\mathrm{CAD}$ systems go through the phases of fashion style design, pattern design, pattern grading and marker making. Some typical commercial softwares of 2D garment

\footnotetext{
* Corresponding author.

E-mail addresses: liuyongjin@tsinghua.edu.cn (Y.-J. Liu), livesforce@yahoo.com (D.-L. Zhang), meymf@ust.hk (M.-F. Yuen).
}

CAD include Toray-Acs in Japan [111], Gerber in United States [112], Investronica in Spain [113], Lectra in France [114].

To release the heavy dependencies on pattern-masters' experience in 2D systems, 3D garment CAD methods with the technologies of human body measurement are studied and proposed as an alternative solution to non-expert users. The key techniques include 3D human body measurement and modeling, 3D garment design on digital human models, 3D draping simulation, and 2D pattern generation from 3D space. Two well known commercial softwares of 3D garment systems are AssystBullmer in German [115] and Dessingsim in Japan [116].

Garment design and simulation are intensively studied in the fields of both computer graphics and computer-aided design. In this paper we present a detailed survey on CAD methods in 3D garment design, showing their promising characteristics and their relationship to $2 \mathrm{D}$ CAD methods. For the state-of-the-art techniques in garment animation and rendering, the reader is referred to the monographs $[47,100]$ and the excellent survey work in $[22,97]$. This survey is partially based on the authors' experience on building the 3D garment CAD system developed at the Hong Kong University of Science and Technology. The second author also co-organized the ACM SIGGRAPH'05 Course Notes [22], from which some material presented here comes. The overall structure of this paper is aimed at presenting a systematic view of development of the 3D garment CAD platform.

This paper is organized as follows. Section 1 covers the introduction of 2D and 3D Garment CAD platforms. Section 2 presents the basic approaches. Section 3 describes the human modeling and Section 4 covers the garment models. Section 5 details the draping methods and Section 6 outlines the 3D to 2D pattern 
transformation. Section 7 reviews the geometric detail modeling approaches and Section 8 summarizes the parallel computation and GPU acceleration techniques. Section 9 provides a perspective for future directions and Section 10 concludes the review.

\section{Basic approaches}

In this section, we outline the basic approaches in 3D garment design. Based on this foundation, in subsequence sections, we will review incremental progresses towards some more advanced CAD methods in garment design.

\subsection{Feature-based human body modeling}

With the advance of 3D scanning techniques, the individualized human body can be easily captured and modeled with a mesh model [1]. In addition to geometric definition of the human model, it is necessary to use semantic features to facilitate the garment design on the model. Assume that the model is in a normal pose (we will review the techniques in Section 3.2 how to release this restriction). In the middle of Fig. 1, two sets of sizing parameters are used to characterize the human body: the heights $h_{i}$ specify a set of cutting planes parallel to a base plane in the model space, and the girths $g_{i}$ are measured on each cutting plane intersecting the human body. The cutting planes are used to build a classification on the vertices, edges and faces in the body mesh model such that each of them is associated with a semantic feature in the set \{head, neck, shoulder, chest, bust, waist, hi p,leg, foot, etc.\} (see the left of Fig. 1). The segmentation of human body model also leads to a natural parameterization of the model which can automatically provide accurate measurement of the individual model (see the right in Fig. 1).

The segmentation process (or in other words, cutting plane determination) usually makes use of some rules of thumb: for example, anthropometry shows that body height equals to eight head tall [82]. The special characteristics of the human body are also helpful to establish the locations of cutting planes. These characteristics include neck points, underarm points, busty points, belly-button point and crotch point, etc. For example the crotch point is the point starting from which the cross-section of the body changes from one circle to two circles. So a simple and effective way to determine crotch point is to cut the body at $5 / 8$ total height from the top [122]. Refer to Fig. 2. In the cross-section, the intersection points are sorted and the maximum gap between two neighboring points (denoted by $P_{l}$ and $P_{r}$ ) separate the left and right legs. At the middle $P_{o}=\left(P_{l}+P_{r}\right) / 2$, a perpendicular plane is setup. The lowest intersection point of this plane with the body is the crotch point. More comprehensive and innovative CAD methods for human body segmentation will be presented in Section 3.

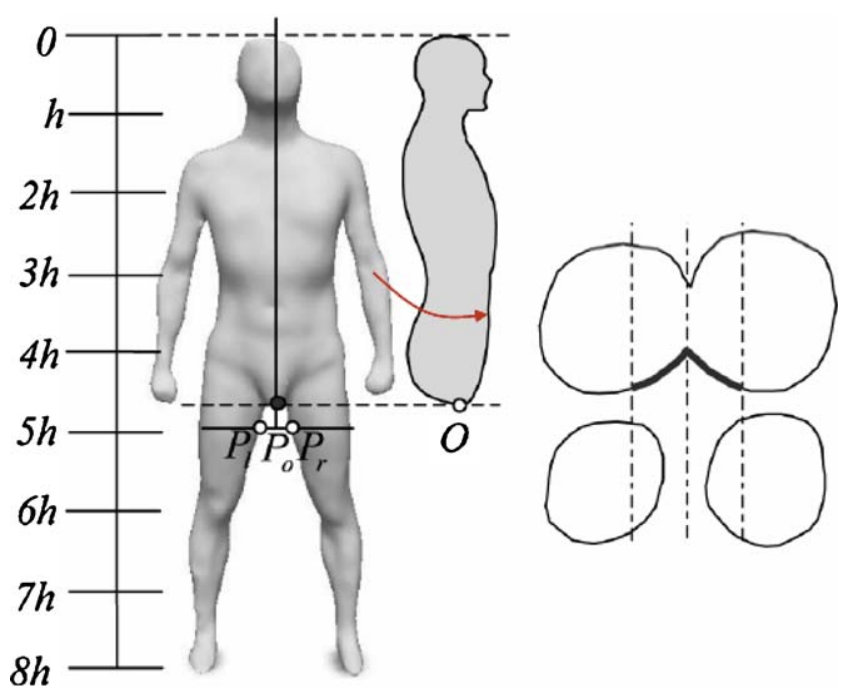

Fig. 2. Crotch point determination [122] (courtesy of Prof. Bugao Xu).

\subsection{D garment design and modification on human model}

Given the human model with detailed semantic features, traditional procedure of a pattern maker can be mimicked by using computers. A case study of ladies' dress design is presented in [74]. Let the human model be oriented such that it stands on the $x y$ plane, the $x z$ plane cut the body into left and right parts, and the $y z$ plane cut into front and back parts. Refer to Fig. 3. The shoulder seam is defined as a line segment connected by points 1 and 2 which is in the intersection of the body and the plane $P_{\text {side }}$ parallel to the $y z$ plane and towards back about $20 \mathrm{~mm}$. The same plane $P_{\text {side }}$ defines the points $3,5,6$ in the side seam of the dress as well. The point 4 is defined on the planar curve of the waist which move forwards in the waist by $20 \mathrm{~mm}$. The points 3,10,18 are on the curve of chest, 4, 9, 19 on the waist and 5, 8, 20 on the hip. I.e., each point is related to a semantic feature on the human body. The closed curves at the crosssections of the chest, waist and hip circumferences of the dress are determined by enlarging the intersection curves. The enlargement determines the tightness and fitness of the dress over the human body. The final dress is made up by adding more auxiliary curves and points which is also determined by rules related to the known points 1-25 in the dress and the semantic human model. Given such a set of deterministic rules, for any customized human model, the corresponding customized dress is determined immediately.

The 3D garment on human body can be further modified using virtual scissor in computer [89]. The widely used tool is provided by OpenGL with the interaction functions. The users can pick point,
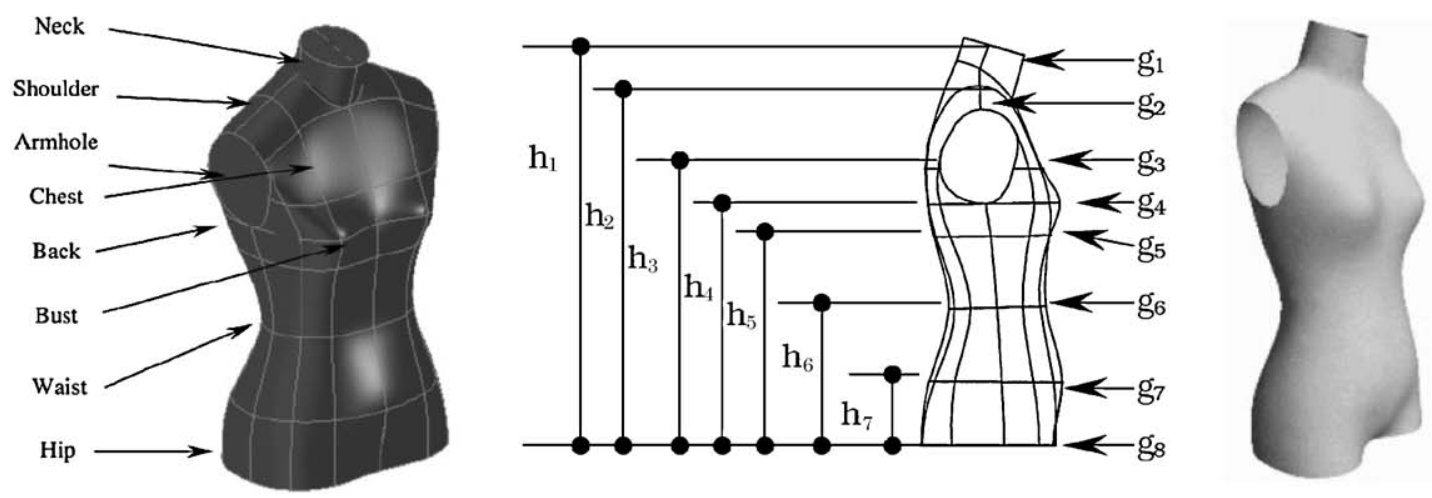

Height $=750 \mathrm{~mm}$

Bust $=861 \mathrm{~mm}$

Waist $=638 \mathrm{~mm}$

Hip $=937.5 \mathrm{~mm}$

Fig. 1. Human body modeling and segmentation with semantic features [6,7] (courtesy of Prof. Matthew Yuen). 

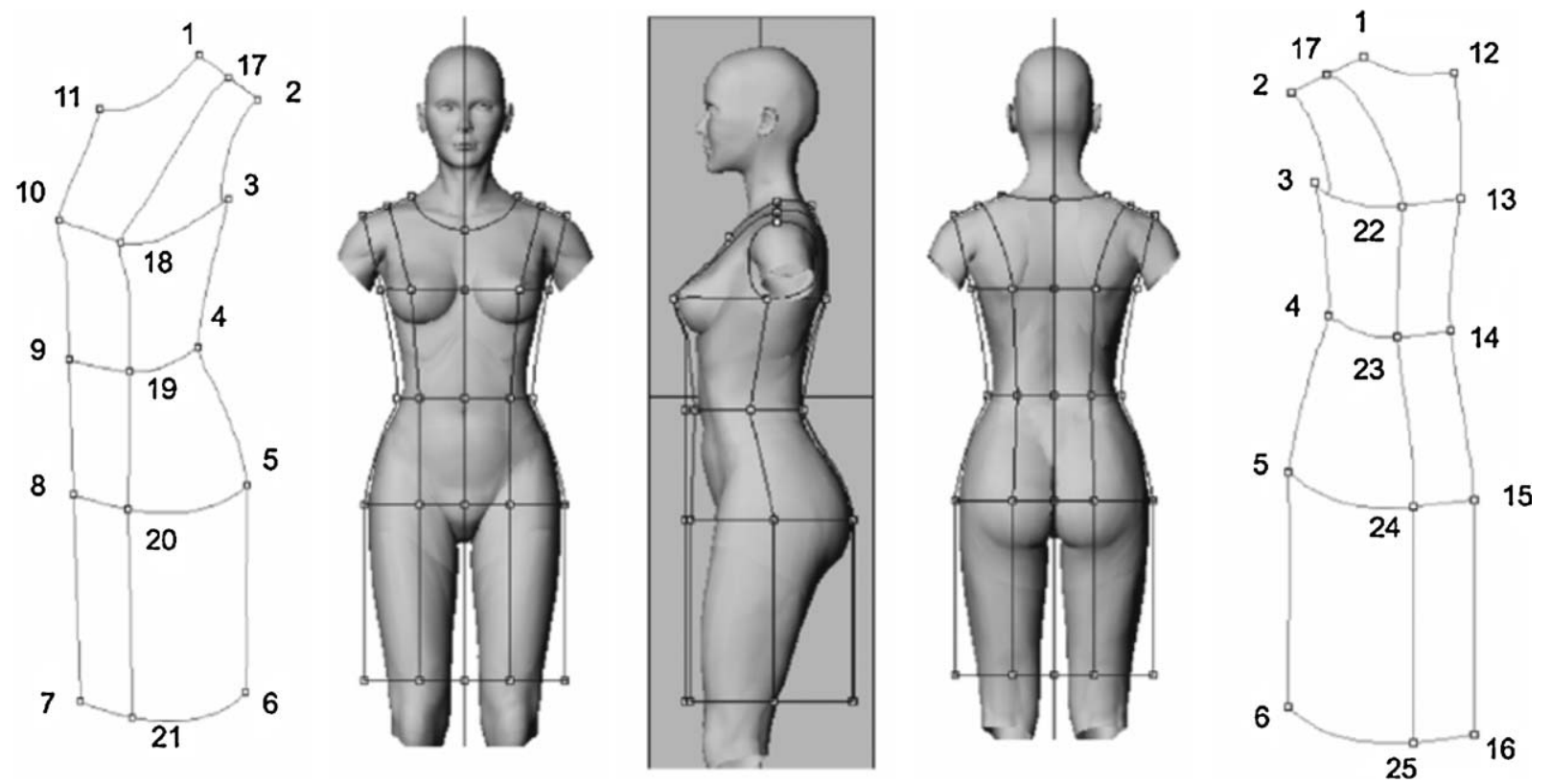

Fig. 3. Ladies' dress design on human model [74] (courtesy of Prof. Slavenka Petrak).

edge and face on the human model and can also draw free-form curves on the human model as well as on the garment. Bases on these operations, virtual scissor can aid the users to make freeform style design over existing garments fitting on the human body. More details are discussed in Section 4.

\subsection{D garment draping and animation}

A garment on human body can be classified into two parts [24,53]: one is the fit part which is in direct contact with the human body and the other is the fashion part which is draped freely to make the aesthetic appearance. The fit part can be directly determined by the semantic features on the human body while the fashion parts are simulated with numerical techniques in which the mechanical material of the garment plays an important role. Many deformable cloth models are proposed for efficient garment draping and animation with which the customers can visualize the realism of virtual try-on $[22,47,97,100]$ (refer to the left of Fig. 4). Deformable cloth models and numerical integration techniques are reviewed in Section 5.

\section{4. $2 D$ pattern generation}

The garment industry eventually needs the 2D patterns to manufacture the garment. Given 3D garments, it should be flattened into a plane without stretch and distortion; in mathematics, this property is characterized by the developable surface [28]. Sometimes a small stretch is allowed in garment flattening. By using the colorindex mapping, computer-aided tools can visualize the stretch by different colors inside the flatten patterns. Usually hot color corresponds to large stretch and cold color to low stretch. Refer to the right of Fig. 4. If the stretch is too big for a piece of garment, cutting lines are needed to release the stretch energy. Section 6 will review the techniques for $3 \mathrm{D}$ to $2 \mathrm{D}$ pattern transformation.

\section{Human modeling}

Human body modeling is a challenging problem with a wide range of applications from virtual surgery to animation in feature movies [67]. With the aid of laser scanners, nowadays, it is easy to obtain the 3D information of individual body in the format of point clouds. Various techniques have been proposed to reconstruct a continuous 2-manifold model from noised point cloud. Notably, the techniques can be typically classified as three types: implicit, parametric and triangulation methods.

The implicit methods describe shapes as level sets of a scalar field $[11,98]$. The seminal work reconstructing implicit surfaces from point cloud includes the signed distance fields $[46,25]$, the level set methods [121], the moving least squares (MLS) methods [2] and the radial basis functions (RBFs) methods [18]. Parametric

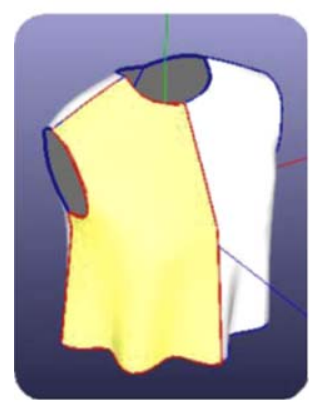

draping

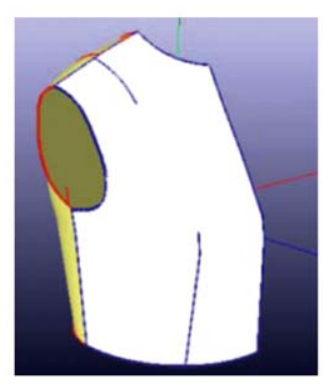

without draping
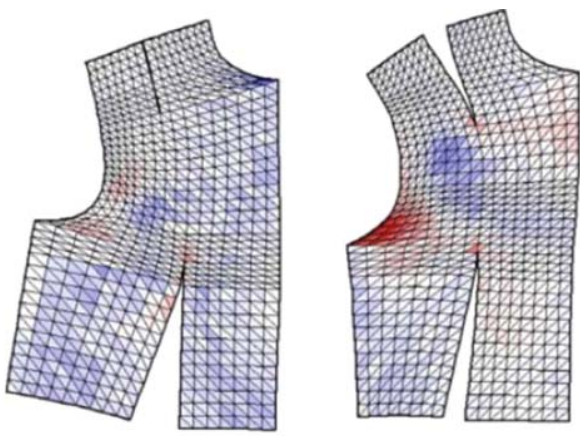

2D flattened pattern with cutting line

Fig. 4. Garment draping and 3D to 2D pattern transformation [53] (courtesy of Prof. Chang Kyu Park). 
methods fit parametric surfaces to the point cloud. NURBS surfaces are most commonly used [37,75], which are best suited for describing shapes with prescribed topological type, mostly homeomorphic to an open disc [32,110]. To overcome the limitation of the regular structure of tensor-product surfaces, Tspline is proposed as a practical solution [83]. Triangulation methods locally connect the data points into a globally structured form of triangular meshes. Usually triangulation methods utilize Delaunay triangulation and its variants $[3,19]$. If the target shape is restricted to human body, an efficient template mesh-fitting method is proposed in [1] which utilizes 3D sparse marker to reconstruct high-resolution human bodies from uncompleted scanned data.

In garment design, in addition to continuous human model reconstruction from measured point cloud, special attentions are paid to build semantic features on the human model so that the garment can be efficient designed and encoded with respect to these features. In [107] a practical and efficient framework is proposed which consists of three phases: data preparation, human model construction and semantic feature extraction (ref. Fig. 5). Assume that the model stands in a standard symmetric pose (in Section 3.2 we will review techniques how to achieve this from arbitrary poses). By auto-locating three key feature points at arm pit and crotch points, the system in [107] first partitions the whole human body into six parts with fixed topology: head root, major body, left arm, right arm, left leg and right leg (ref. Fig. 6). The feature points can be robustly found by monitoring the topology changes of the closed circles at sequential cross-sections (ref. Fig. 2 for crotch point detection). To build the complete semantic features over the human model, three more key features - neck, bust and belly button points - need to be specified. The rules of thumbs are as follows. The neck feature point is the extreme point around $7 / 8$ height of the human model from the silhouette of the front view. The bust point is the extreme point around 3/4 height from the silhouette of the right view. The belly button point is around 5/8 height from the silhouette of the right view. Given these necessary six features (plus arm pit and crotch points), the whole semantic feature net is established by proportion rules used in the fashion industry $[88,92]$.

Without inferring continuous model from point cloud, Kim and Kang [52] propose a similar technique which directly generates garment patterns from body scan data. However, we recommend to first build the continuous model of human body with consistent parameterization. The meaning of consistent is related to the common set of semantic features and we will discuss more details in Section 3.3. The advantage is that after the garment template is designed on a standard size of mannequin and is encoded with the

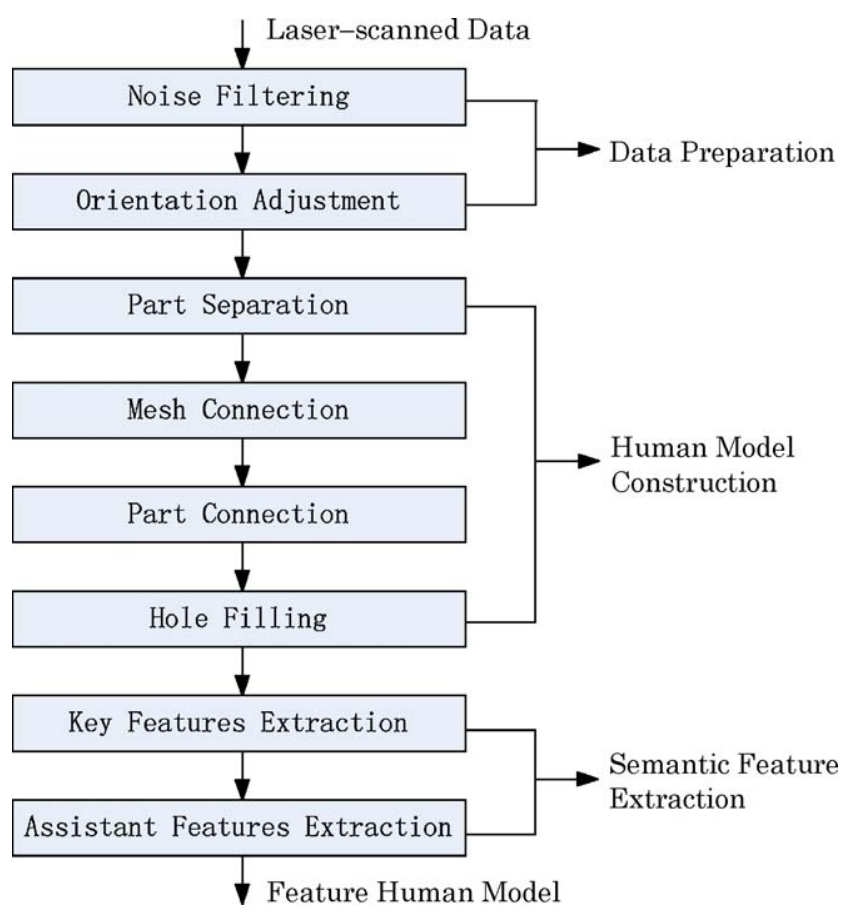

Fig. 5. Overview of the reconstruction system of human body proposed in [107].

semantic features of human body, the garment pattern can be immediately transferred and refitted to any customized human body. Thus one of the major challenges in 2D garment CAD systems, automatic made-to-measure, is efficiently solved in $3 \mathrm{D}$ space.

\subsection{Other sources of individuals' information}

Laser scanning is not the only source to obtain the customized body information. Given the consistent parameterization of human body models, two other popular methods are based on photographs and size charts. Photo-based methods take photographs of a human body from orthogonal views, i.e., front view and side views. The rich research in image process allows us to efficiently extract the silhouette of human body from photographs [76]. By establishing the 2D features of human body and its associated one-to-one mapping to the 3D semantic features in a generic human model, Lee et al. [58] and Wang et al. [108] propose efficient view-dependent deformation to drive the generic 3D model into
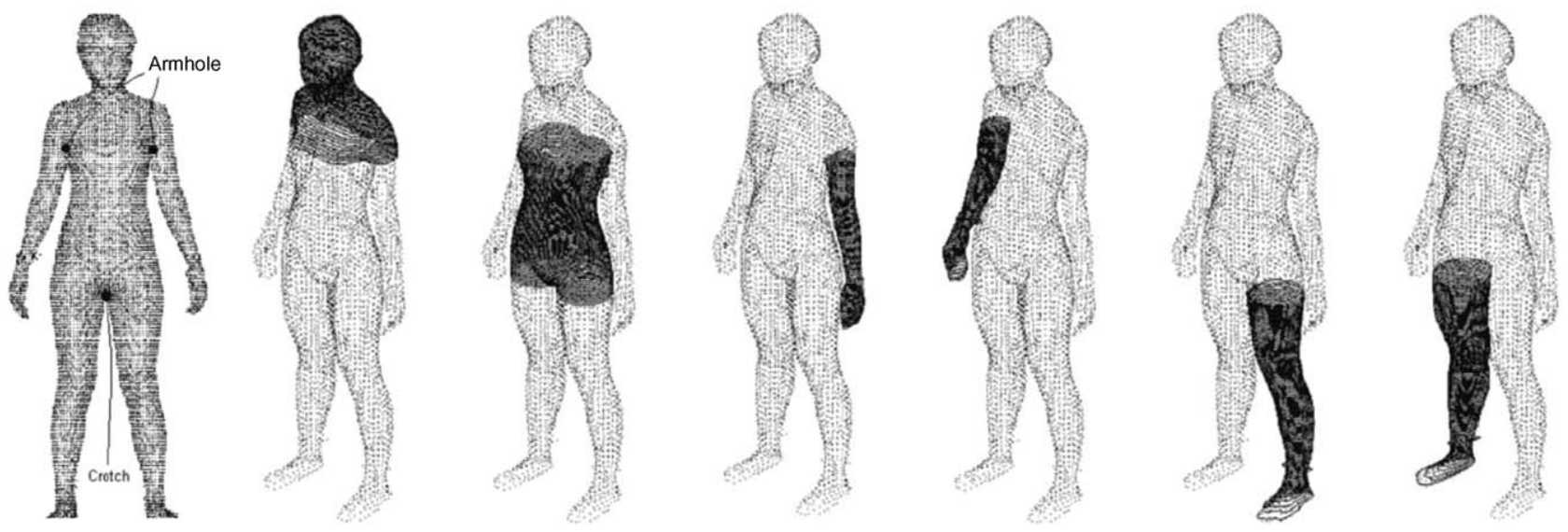

Fig. 6. Three key feature points (two arm pit and one crotch points) and the associated partitions with six semantic parts [107] (courtesy of Prof. Charlie C.L. Wang). 
individuals. Lee's method [58] uses the 2D skeleton features of the human body in the photographs, while Wang et al. [108] establishes a set of consistent rules to automatically locate the 2D feature points in the silhouette.

The size-chart methods enable the user to create his/her own virtual model by inputting a number of sizing parameters. This is particular useful for remote design through web interfaces. A representative size-chart method is proposed in [84]. In this method, a database of 3D scanned data of human body models is stored in the server. When the user inputs some sizing parameters via internet at the client end, the information is transferred to the server where several human models with similar parameters are chosen first. Then by interpolating these existing human models in the geometric space using radial basis functions, a new model satisfying input size parameters is created and transferred back to the client end.

\subsection{Standard pose adjustment}

In the above sections, the human body is assumed to be at standard symmetric pose. The real human bodies under measurement, however, are always in different poses. So it is necessary to have CAD techniques to auto-correct these different poses into a standard one. In [102] a pragmatic approach is proposed to make human model symmetric. Denote the original asymmetric model by $\mathrm{M}^{+}$and locate it in the coordinate system whose origin is at the crotch point and $x z$ plane cut the model into left and right parts. By finding the mirror position of each vertex in $M^{+}$according to $x z$ plane, a mirror mesh $M^{-}$is obtained. The symmetric model is built by the interpolation $M^{S}=\left(M^{+}+M^{-}\right) / 2$. The features $F$ associated with $M$ is obtained in the same way: $F^{s}=\left(F^{+}+F^{-}\right) / 2$. For human models with approximate standard poses, this method works pretty well.

For objects with arbitrary different poses far from symmetry, a rigorous technique is proposed in [71] to enhance symmetries of a model while minimally altering its shape. The method models the symmetry as a deformation process. By mapping compatible sample pairs into the space of reflective transformations, the deformation can be viewed as an optimization process in which an elegant closed-form solution is formulated. Besides the application of body measurement in the garment design, various applications of symmetrizing deformation in design and shape analysis are also shown in [71].

\subsection{Feature modeling}

Two key CAD methodologies are widely used in 3D garment systems: feature recognition and design by features [16]. Feature recognition methodology [60] recognizes user-specific features from a geometric model, such as human bodies, according to the feature templates predefined in a feature library. Given the necessary features on human bodies, the design by feature methodology [27] creates the feature model of garment by composing the available features in a feature library.

In the most abstract level, objects in engineering applications are categorized into two distinct classes: regular shaped objects and sculptured objects. Human body is a typical sculptured object. A semantic-oriented feature model technique based on formal languages is proposed in [7] for sculptured objects. As shown in Fig. 7, the semantic feature model of an object consists of three level of abstraction: object level, feature level and geometry level. The configuration of a sculptured object is represented by a feature graph $G_{o b j}=(V, E)$. The graph is formalized by a feature language which consists of two components: vocabulary and grammar. In $G_{o b j}$, both vertices and edges are vocabularies. The grammar is a set of production rules manipulating the vocabularies. For human

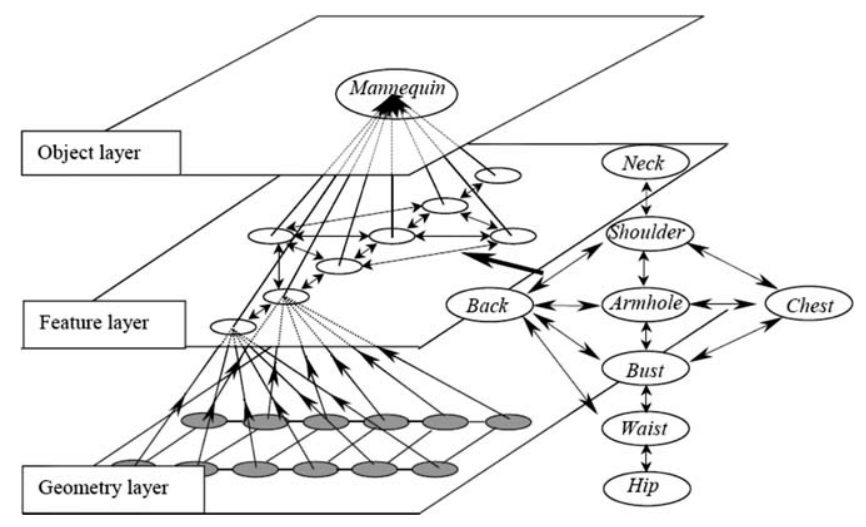

Fig. 7. The feature model of a mannequin shape [7] (courtesy of Prof. Matthew Yuen).

body, the vocabularies are defined by a constituent features set $F_{\text {human }}=\{$ neck, shoulder, chest, back, bust, armhole, waist, hi $p\}$ and a set of dimensions $D_{\text {human }}=\left\{h_{j}, g_{j} \mid i=1, \ldots, 7\right.$ and $\left.j=1, \ldots, 8\right\}$. See Fig. 1 for an illustration of the dimensions $D_{\text {human }}$. The used feature language grammar is a simple string grammar [38]. Compared to the geometry-oriented approaches for describing sculptured objects such as human body, the semantic feature modeling technique uses geometry as only a set of auxiliary information embedded in the feature vocabularies. The biggest advantage is that the sculptured objects can be modeled and operated at a consistent topological level, disregarding of the potentially infinite possible shapes, and thus, providing a systematic representation scheme of the object that can be used in various engineering applications domains.

\subsection{Parametric design of human models}

Towards the geometric realization of the topological abstract model of human body, a novel feature based parameterization approach is proposed in [102]. Based on the parameterization, the sizing dimensions can be easily obtained through semantic features. First five key features - neck, underarm, busty, bellybutton, crotch points - are extracted using the rules of thumb stated in [107]; among them, three features are illustrated in Fig. 6. See also Fig. 8a. Given these key feature points, the complete set of features $[7,107]$ can be realized using the cutting planes and the projection planes (ref. Figs. 1 and $8 \mathrm{~b}$ ). Note that the abstract topological structures of these features on individuals' body data are all the same as shown in Fig. 7[6,7]. The geometric realization of the feature model has three levels of entities: feature points, feature curves and feature patches. According to the feature definition, the feature points can be classified and organized into feature curves. The sizing parameters of human body are given by feature curves. To encode garment design features into a mannequin with standard sizes and for automatic made-tomeasure based on individuals' sizing parameters, it is necessary to incorporate feature patches into the feature human model [106]. In [102] the feature patches are spanned by Gregory patches which interpolate generally $n$-side feature curves as the boundaries [42]. By interpolating both positions and tangent vectors, the final body surface is $G^{1}$-continuous (ref. Fig. 8d).

Given a large database $D$ of human bodies with a consistent parameterization, new individuals' bodies can be generated according to the specified sizing dimensions by synthesizing example models in $D$. Refer to Fig. 8e. This technique, called parametric design of human model in [102], falls into a general category well-known in computer graphics as example-based model synthesis $[40,84,85,87]$. Denote the $n$ human bodies in $D$ by $H_{i}$, $i=1,2, \ldots, n$. The synthesized body $H_{s}$ is obtained by interpolation 


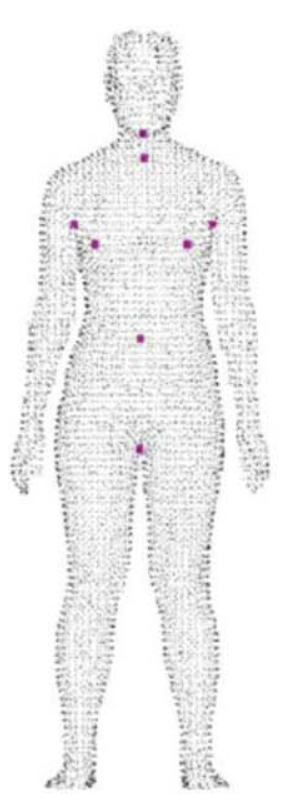

(a) Key feature points

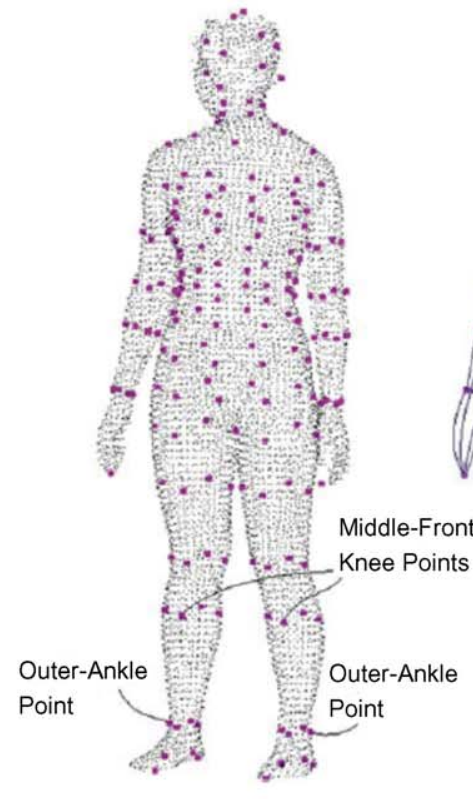

(b) The complete set of feature points

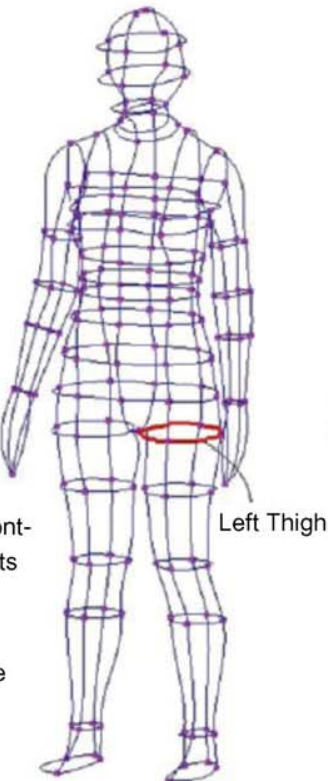

(c) Feature graph

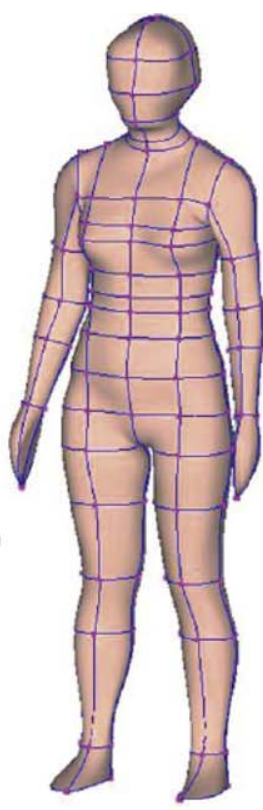

(d) Feature patches on human body

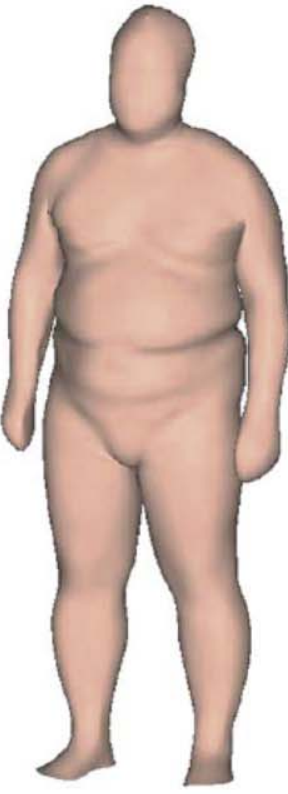

(e) Modification by sizing dimension

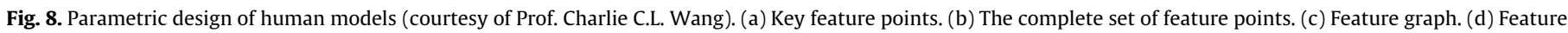
patches on human body. (e) Modification by sizing dimension.

as $H_{s}=I\left(w_{1} H_{0}, w_{2} H_{2}, \ldots, w_{n} H_{n}\right)$, where $I(\cdots)$ is the interpolation function based on the feature graph, the weights form partition of unity $\sum_{i=1}^{n} w_{i}=1$ and $w_{i} \geq 0$. Given a human body $H$ with featurebased parameterization, its dimension vector is indicated by the feature graph and is extracted by a measurement function $\Psi(H)$. Then, given any reasonable input sizing dimension vector $D$, The corresponding individuals' body is built by seeking the optimal solution to minimize the objective function $\min \left\|\Psi\left(H_{S}(X)\right)-D\right\|$, where $X$ is the parameter vector to be optimized which consists of scaling and weighting coefficients. Conjugate gradient method is used in [102] to optimize the solution.

\subsection{Limitations of existing methods}

There are two cruxes in the existing methods for human modeling, which offer the technical challenges for the future work. The first crux is related to the acquisition of body dimensions. In all acquisition methods including laser scanning and photography, the test subjects must wear tight clothes to show the body profile. To relax the customers, can the body dimensions be automatically measured with sportswear or skirts? Thermal radiation measurement methods such as infrared imaging techniques may contribute in this direction.

The second crux lies on the automatic feature detection. Although many rules of thumb exist as we summarized in previous sections, eventually intensive human intervention is required to fine tune accurate locations of necessary feature points. Stable and robust automatic methods are solicited in this direction.

\section{Garment design}

Based on the feature-oriented parameterization of the human body, design-by-feature methodology $[16,27]$ is applied to construct diverse feature-based profile templates of garments, each of which represents a different product styling of garment. The garment feature template is directly related to the feature graph of a parameterized human model by incorporating easing relationships [106]. When the body model is modified by inputting sizing dimensions, the easing relationship will automatically rescale and fit the garment product well on the individual body. Thus the difficult, experience-based 2D grading problem is avoided in 3D garment CAD systems.

The garment feature template consists of feature nodes, profile curves and surface refinement rules (ref. Fig. 9). Each feature node is encoded with a reference element in the featured human model (ref. Figs. $8 \mathrm{~d}$ and $9 \mathrm{~b}$ ). The reference element can be vertex, edge or face in the body model. In each reference element ref, a consistent local frame can be uniquely determined: the frame consists of an origin $\mathbf{0}$ and three linear independent vectors $\mathbf{t}_{1}, \mathbf{t}_{2}, \mathbf{n}_{3}$. Given the frame re $\mathbf{f}\left\{\mathbf{O}, \mathbf{t}_{1}, \mathbf{t}_{2}, \mathbf{n}_{3}\right\}$, the feature node is encoded by a displacement vector $\mathbf{f n}\left\{d_{1}, d_{2}, d_{3}\right\}$ and its position in the global coordinate system can be decoded by $\mathbf{f n}\left\{x_{1}, x_{2}, x_{3}\right\}=\left\{\mathbf{O}+d_{1} \mathbf{t}_{1}, \mathbf{O}+d_{2} \mathbf{t}_{2}, \mathbf{O}+d_{3} \mathbf{n}_{3}\right\}$. Given an individual body model, its element's position may be changed to $\operatorname{ref}^{\prime}\left\{\mathbf{O}^{\prime}, \mathbf{t}_{1}^{\prime}, \mathbf{t}_{2}^{\prime}, \mathbf{n}_{3}^{\prime}\right\}$. Then the new position of the corresponding feature node in the garment template is changed accordingly to $\mathbf{f n}\left\{x_{1}, x_{2}, x_{3}\right\}=\left\{\mathbf{O}^{\prime}+d_{1} \mathbf{t}_{1}^{\prime}, \mathbf{O}^{\prime}+d_{2} \mathbf{t}_{2}^{\prime}, \mathbf{O}^{\prime}+d_{3} \mathbf{n}_{3}^{\prime}\right\}$ (ref. Fig. 9e).

The profile curves in garment feature template are used to control the local shape of final garment style (ref. Fig. 9d). A profile curve is a parametric curve $C_{p}(u)$ associated with an edge $E_{p}$ in the topological graph of the human body. In [106] fourth-order Bezier curves are used. Each profile curve $C_{p}(u)$ is encoded by the displacement curve $e_{p}(u)=C_{p}(u)-E_{p}(u)$ and when an individual body model is used, the new profile curve can be decoded by $C_{p}^{\prime}(u)=e_{p}(u)+E_{p}^{\prime}(u)$ (ref. Fig. 9e).

The surface refinement rules provide details and smoothness on the shape representation of an apparel product. Different interpolatory subdivision rules [123] can be applied here: notably the modified variational subdivision [55] and butterfly subdivision [30] are most popular. Fig. 9f shows an example using the modified variational subdivision rules.

Note that it is important to encode the feature nodes and profile curves in a local frame associated with the underlying model 


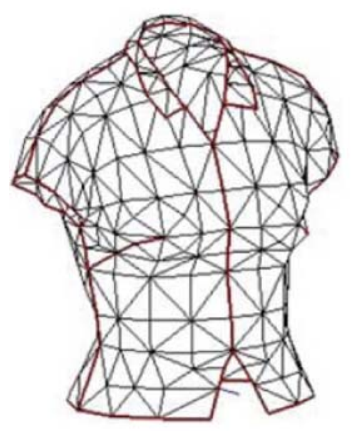

(a) A garment feature template

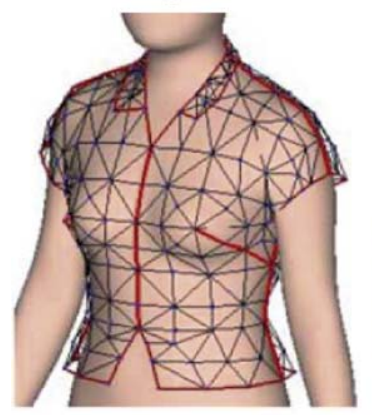

(d) Another view of (c) with profile curves

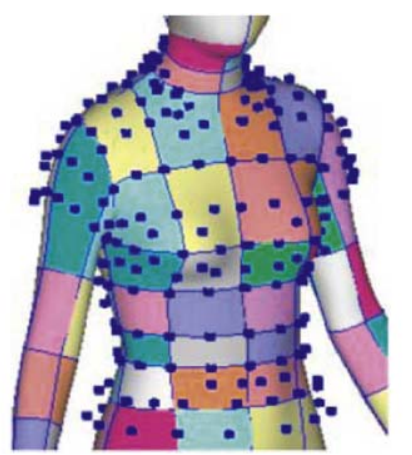

(b) Feature nodes

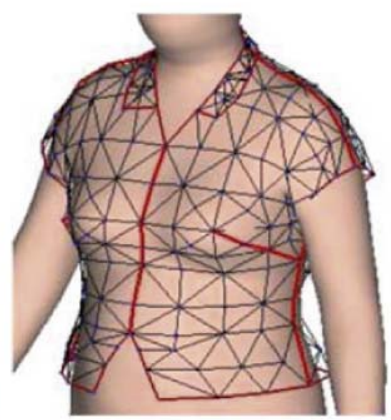

(e) Garment decoding on an individual body

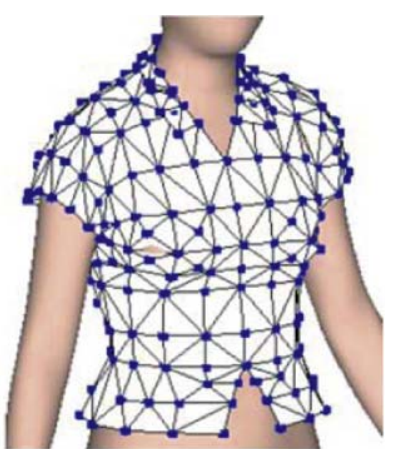

(c) Connectivity of feature nodes

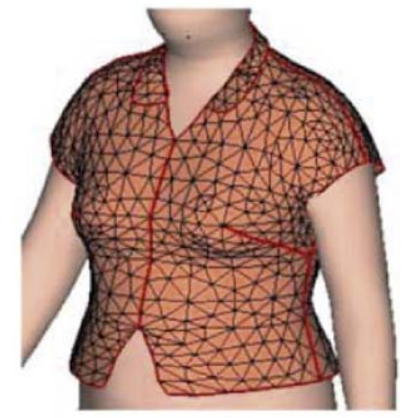

(f) Subdivision refinement

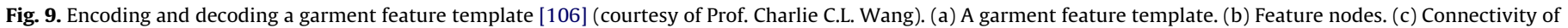
feature nodes. (d) Another view of (c) with profile curves. (e) Garment decoding on an individual body. (f) Subdivision refinement.

(human model in the case of garment design), so that the following advantages can be achieved:

1. Detail preserving. By encoding in local frames, high-frequency details on the garment are separated from the basic shape. When the underlying body model is changed, the basic shape of the garment will also be changed but the details will be decoded and faithfully reconstructed via local frames.

2. Smoothness. Since subdivision surfaces usually generate $C^{1}$ or $C^{2}$ limit surfaces, when the human body model is changed, the free form surfaces of the associated garments will be changed smoothly.

Similar techniques of detail-preserving modification and animation have been widely studied in computer graphics with general graphics models. Lee et al. [59] and Guskov et al. [43] encoded the complex graphics models using a basic mesh together with a scalar detail set. The transformation is demonstrated to be very smooth. Liu et al. [65] improved the method in [59] by using a multi-level displacement field.

A typical commercial CAD system. LookStailorX [116] is a 3D garment design system that uses featured human models and garment feature templates. It creates a 3D base garment automatically from a human body. For the human body, it can be a predefined mannequin specially for garment design, or a scanned body captured by a body scanner. To create the 3D base garment, firstly, the contour lines are generated automatically according to feature points of the human body. Then the surface of the 3D garment is created from its contour lines, as shown in Fig. 10. The contour lines can be edited interactively, and the garment shape will be modified accordingly. The base garment is used for creating 2D patterns of the final garment to be designed. The method of developing 2D patterns of the system will be reviewed in Section 6.

\subsection{D pattern input, virtual sewing and fitting}

Since 2D garment CAD systems have been widely used in industry, it is desired to provide application programming interface (API) functions in 3D garment CAD systems for making use of the traditional 2D pattern designs. Given such 2D pattern input functions, the necessary operators to convert the design to $3 \mathrm{D}$ are virtually sewing and fitting on an individual body model.

In a 3D computer-aided garment design system [72], triangulated cloth patterns are used and mapped onto a mannequin model by minimizing a quadratic energy function. Aono et al. [5] gave a further study on the fitting problem. In [96] a versatile and efficient technique is proposed to simulate $3 \mathrm{D}$ dressing results from 2D pattern input. Usually the 2D-to-3D transformation is treated as an elastic deformation process with large displacement but small stretch deformation. In [36], a physical based deformable model is proposed for 2D-to-3D sewing and fitting. In this model, the cloth patterns are modeled by a triangular mesh composed of mass nodes and massless edges: edges works as springs. Both structural and shear springs are defined as shown in Fig. 11. A similar massspring model is proposed in [79].

Lagrange mechanism is used in [36] to drive the sewing and fitting process. Each node position is determined by the motion of equations:

$\mu \frac{\partial^{2} X}{\partial t^{2}}+\gamma \frac{\partial X}{\partial t}+\delta \varepsilon=f$

where $X$ is the position vector of the nodes, $\mu$ is the nodal mass and $\gamma$ are the damping factor. $\delta \varepsilon$ is the internal elastic force derived from the variations in the tensile energy. $f$ is the external force which includes sewing forces and collision reaction forces. The sewing forces pull and sew different patterns together. The 


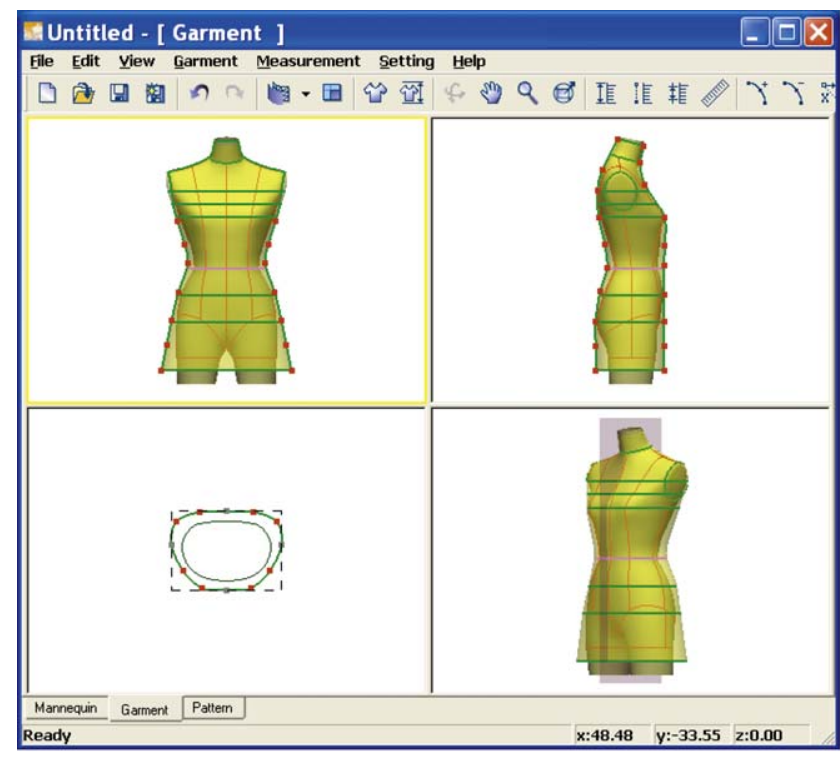

System overview
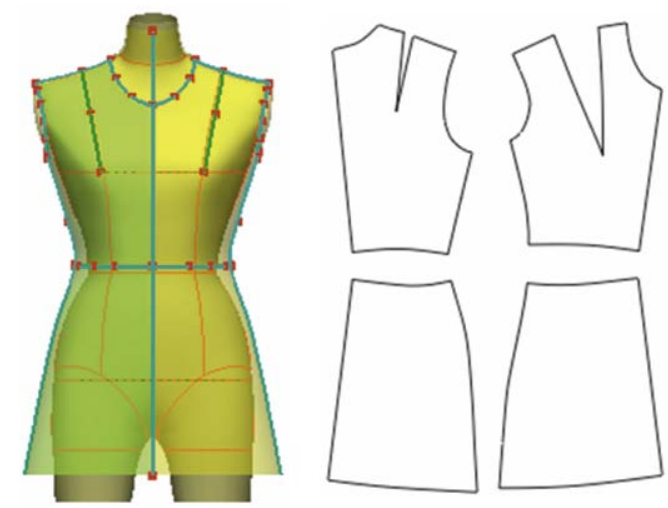

Flattened 2D patterns

Base garment

with editable

contour lines

Fig. 10. A 3D garment design system LookStailorX developed by Digital Fashion Ltd, Japan [116] (courtesy of Dr. Dong-Liang Zhang).

collision reaction forces penalize cloth patterns from penetrating into the body or other cloth patterns. The forward Euler method is used in [36] to numerically solve the Lagrange equation of motion.

\subsection{Sketching input}

One advantage inherent in 3D garment systems is that the users can directly make their design in 3D space without through the intermediate media of $2 \mathrm{D}$ patterns. 3D sketch is proposed as a fundamental tool to make such 3D design available [105]. Recall that the garment feature template consists of feature nodes and profile curves. Given the human body model, the feature nodes can be specified by the picking function provided by OpenGL APIs. Its encoding with elements in human models is established at the same time. 3D profiles of a piece of garment are specified by 2D interactive sketches. Each 2D stroke drawn by users on the screen actually determines a cutting plane through the projection direction (see Fig. 12a). By intersecting this plane with the human model and projecting the nearly vertices onto the plane, the 3D sketch on the human model is obtained. To sketch profiles, the easing space between the garment and the human model is also provided by the users.

Each sketched profile is presented as a point list: each of points is encoded locally with an edge in the topological graph of the human model. To highlight the points in the sketched profile using 2D small circles, the users can further pick and drag these small circles to make further modification of their design. During the drag modification of garment profiles, the updating on the local encoding with the underlying human model must be carefully handled. A novel updating scheme is proposed in [105] by using a least square optimization method.

In computer-aided design and computer graphics, free-form 3D object design through 2D sketches on screen has been studied over the years. The SKETCH system [119] introduces a gesture-based interface and utilizes CSG operations to build solid models. The Teddy system [49] constructs a rounded freeform solid by sweeping along the medial-axis-like skeleton with adaptive radius. Recently the Teddy system is extended to make animation available by moving the 2D sketches [48]. In [95] an intuitive sketching system is proposed to design

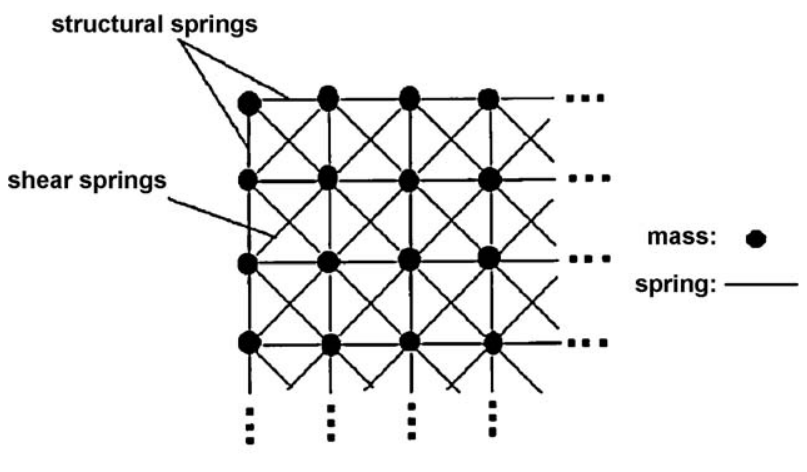

A spring-mass system
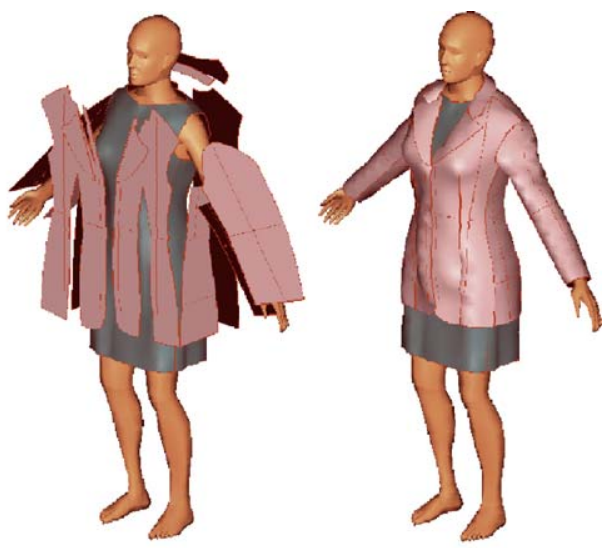

2D-to-3D transformation

Fig. 11. Virtual sewing and fitting with 2D pattern input. 


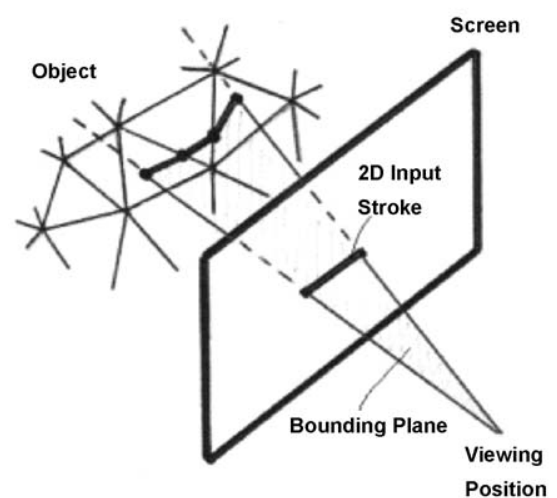

(a) Mesh painting
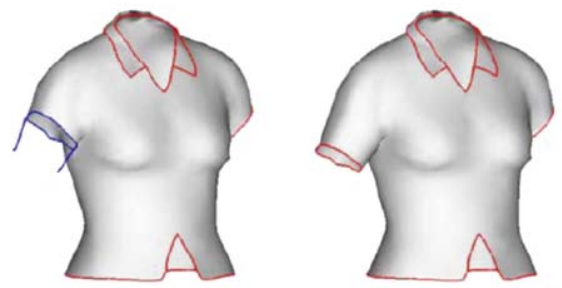

(c) Mesh extrusion
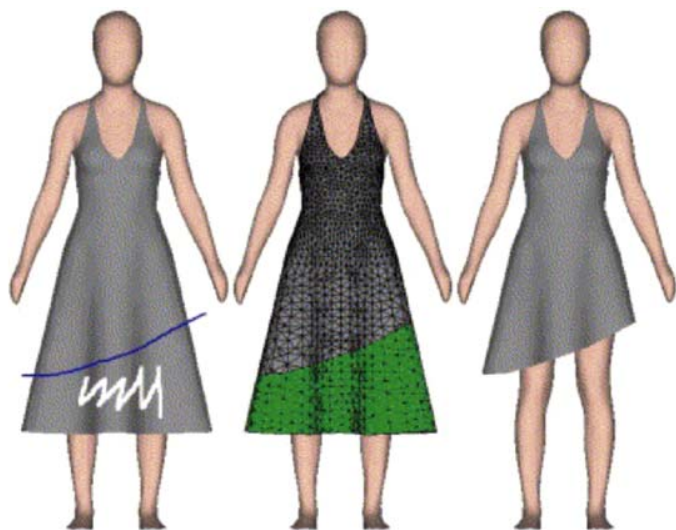

(b) Mesh cutting
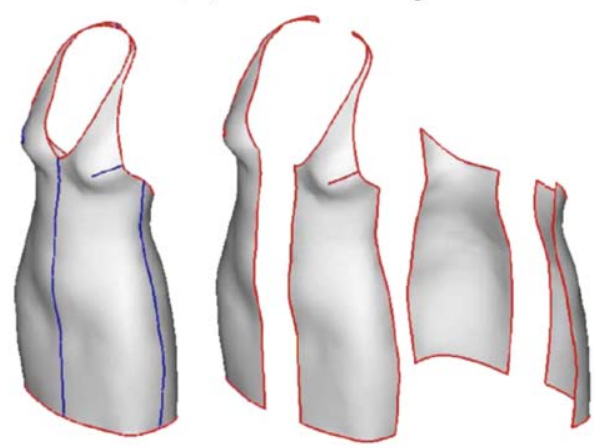

(d) Mesh partition

Fig. 12. 3D modification tools based 2D sketches (courtesy of Prof. Charlie C.L. Wang). (a) Mesh painting. (b) Mesh cutting. (c) Mesh extrusion. (d) Mesh partition.

garment using contour lines. This sketching system is further used in [26] for garment design towards a fast geometric approach.

\subsection{D modification}

3D sketch is also a useful tool for free-form modification of garment template styles. In [106] four sketching-based modification operators are proposed: mesh painting, mesh cutting, mesh partitioning and mesh extrusion. Referring to Fig. 12, mesh painting operator creates auxiliary 3D line segments by projecting 2D strokes onto the surface mesh along the view direction. New features can be defined and associated with the new created auxiliary 3D curves. Mesh cutting and partitioning operators are based on painting. Mesh cutting removes some parts from the mesh surface with the aid of $2 \mathrm{D}$ input strokes, while mesh partitioning separates the mesh surface into several pieces by 2D input strokes. Mesh extrusion creates new mesh parts from line segments on the base surface along the input extruding strokes. Users are also allowed to sketch 2D strokes to extrude a surface from the given mesh. This operator is particularly important when designing collars, epaulets and sleeves, etc., where typically non-manifold structure is resulted in.

More comprehensive 3D modification tools are proposed in the literature of both CAD and computer graphics. Suzuki et al. [90] proposed a 3D mesh dragging method for intuitive geometric modeling of free-form mesh models. The mesh painting, cutting and extrusion operators are also used in the Teddy system for toy design [49]. Input 2D strokes can also be used as local skeletons to drive a view-dependent deformation of an arbitrary free-form geometry [81].

\subsection{Limitations of existing methods}

Existing methods design and model garments of simple styles pretty well. However, when the complexity of fashion increases, special attentions must be paid to the fine details such as dartspleats and gathers, etc. In Section 7, we summarize some promising methods of geometric detail modeling from computer graphics literature that could make an impact in this direction.

One more difficulty in the existing methods is how to accurately modify the 2D patterns when human body size and 3D patterns changes. Given the feature relation between the human bodies and 3D garment templates, the modification of 3D garment with individual body size is readily to obtain. However, since there is no direct relation with 2D patterns, each time a body size is changed (so is its related 3D garment), a complete flattening process needs to be performed for $2 \mathrm{D}$ pattern generation. This makes the interactive design process unnecessary long and tedious.

\section{Draping}

Garment is a special soft product whose fitting can only be judged when it is fitted onto a moving human body. Cloth draping refers to the techniques which compute the rest state of a piece of cloth when subjected to a gravitational force field [100]. The early work uses elastic models to compute the simulation of simple scenes such as wind-blowing flags and rectangle cloth draping [93]. Realistic simulation of comprehensive garment is first studied by the research group of Prof. Nadia Thalmann [17,96]. State-ofthe-art garment simulation techniques can be sorted into three classes: geometric based, physical based and hybrid models.

Geometrically based methods model complex geometric details, such as wrinkles and folds of fabrics, using mathematic 
descriptions [45,109]. Recent advances in geometric methods can make realistic fine details in almost real time [26,39]. During the last decade, the methods using the principles of mechanics have attracted much interest and a diversity of physical models are proposed, e.g., the mass-spring models [79,36], the particle models $[13,14,31]$ and the elasticity-based models $[4,33,93]$. A classic work in this class is presented by Baraff and Witkin [9] in which a computational framework for cloth simulation based on an implicit integration method with a large time step is proposed. Physical based methods have solid foundation in computational mechanics and the parameters usually have intuitive physical meaning with the merit that tuning them is not as difficult as the geometric methods. However, its computational load is much higher than the geometric methods. By taking combined advantages of the speed in geometrical methods and the accuracy in physical methods, hybrid methods are proposed [57,44,101].

To generate realistic draping, deformable cloth models usually take into account material properties of different fabric patterns such as cotton, woolen, silk, leather, polyester and chemical fiber, etc. During the simulation process, gravity and collisions need also to take into account to generate adequate folds and wrinkles. In the follows subsections, we summarize them in turn to investigate the state-of-the-art techniques.

\subsection{Deformable models}

Compared to the mass-spring system $[36,79]$ as shown in Fig. 11, the particle systems model the cloth as a collection of particles that represent the crossing points of weft threads; here the cloth pattern is treated as an interwoven grid of threads. In [13] a particle system is proposed that represents the various threadlevel structural constraints using energy function minimization. The constraints are imposed in a local neighborhood of each particle that captures simple geometric relationships of the interwoven grids. As shown in Fig. 13, the energy function of each particle $i$ in particle system consists of five parts: $U_{i}=U_{\text {repeli }}+U_{\text {strechi }}+U_{\text {bendi }}+U_{\text {trellisi }}+U_{\text {gra }} . U_{\text {gra }}$ is the potential energy due to gravity. $U_{\text {repeli }}$ is repulsion energy (Fig. 13Ia) which keeps every two closed particles at a minimum distance and thus gives some measure of collision detection. $U_{\text {strechi }}$ is calculated between each particle and its four neighbors (Fig. 13Ib) which counts for thread stretching. $U_{\text {bendi }}$ measures the energy due to threads bending out of the local plane. $U_{\text {trellisi }}$ is the energy due to shearing around a thread crossing in the plane. To simulate the cloth draping, the total energy $U=\sum_{i} U_{i}$ is minimized over a series of small discrete time step. Experimental results with the Kawabata evaluation system [51] are presented in [14] which shows that accurate large-scale draping behavior of specific types of cloth can be achieved in this model.

The finite element method (FEM) is a classic numerical method in continuous solid mechanics. In [33] finite element modeling of flexible fabric patterns is proposed. FEM uses shape functions (bilinear, trilinear, quadrilinear, or higher order) to interpolate a discrete net to model the continuous cloth surface. From mechanic properties of the material, a Lagrange functional is formulated and minimized using Hamilton's principle to drive the deformation process. Although FEM has a pretty physical meaning of its control parameters, a large sparse linear system needs to assemble and compute at each time step, and thus, it is time-consuming and can not be used in any interactive garment CAD system.

To offer a good compromise between the speed and accuracy, a hybrid model with stable implicit numerical scheme is proposed in [101] which is further adapted in a garment system proposed in [97]. The model describes the accurate surface deformation using a triangular mesh equivalent to a first-order finite element description. Accurate mechanical properties such as warp, bending, shearing, viscoelasticity are specified in the particles while a triangular mesh provides an overall structure of the particles such that implicit numerical integration is possible. One additional merit of this hybrid model is that the triangular mesh in arbitrary connection is used to eliminate topological restrictions of rectangular meshes as in mass-spring models, and thus can flexibly model any shaped patterns.

\subsection{Material models}

Most advanced deformable cloth models can generate visually pleasing draping behavior. In garment industry, however, it is more desired to describe accurate cloth models by simulating the main material properties of fabric. To methodically query the material properties of cloth sample, Kawabata evaluation system (KES) serves this need [51]. As shown in Fig. 14, KES is a standard set of fabric measuring equipments that measure the cloth sample in terms of the bending, shearing and tensile properties, as well as the surface roughness and compressibility. For bending, shearing and tensile properties, a fabric sample of standard size $(20 \mathrm{~cm} \times 1 \mathrm{~cm}$ for bending and $20 \mathrm{~cm} \times 5 \mathrm{~cm}$ for shearing) is tested with the equipment. Forces or moments are plotted as a function of measured geometric deformation: for example, bending and shearing properties of samples of 100\% cotton, $100 \%$ wool and cotton-polyester are shown in Fig. 15. For computational purpose, in [14] linear and quadratic segments are adopted to approximate the original plots using the standard interpolation techniques.

The theory of elastic bending beams states that the strain energy due to bending moment $M$ in an infinitesimal beam $d S$ is $d U=k M d S$, where $k$ is the curvature of beam shape. If the particle system in [14] is used, the bending energy of a particle is $U_{\text {bendi }}=k M \sigma / 2$, where $\sigma$ is the distance of the particle to its neighbors. Similarly, the shearing energy due to the force $F$ on a particle is $U_{\text {trellisi }}=\lim F \cos (\phi) \sigma d \phi$, where $\phi$ is the shear angle defined in [47].

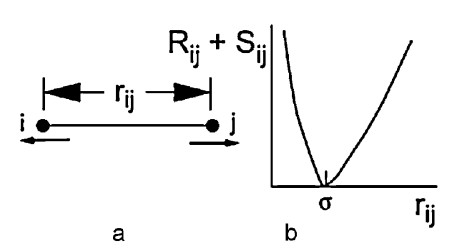

a

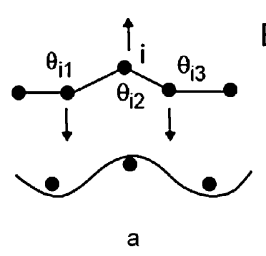

a

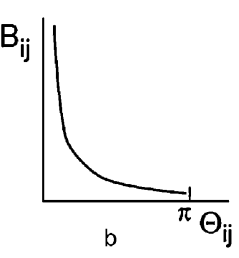

II. Bending
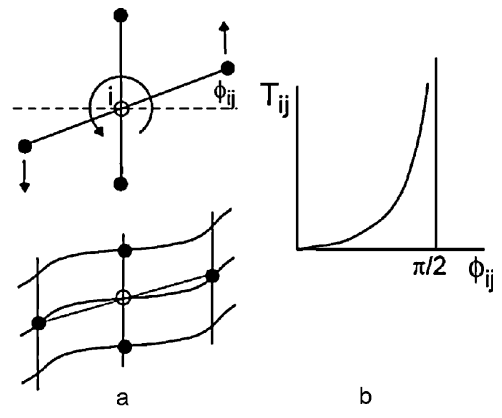

III. Trellising

I. Collision and stretching

Fig. 13. The energy function in a particle system proposed in [13]. I. Collision and stretching. II. Bending. III. Trellising. 

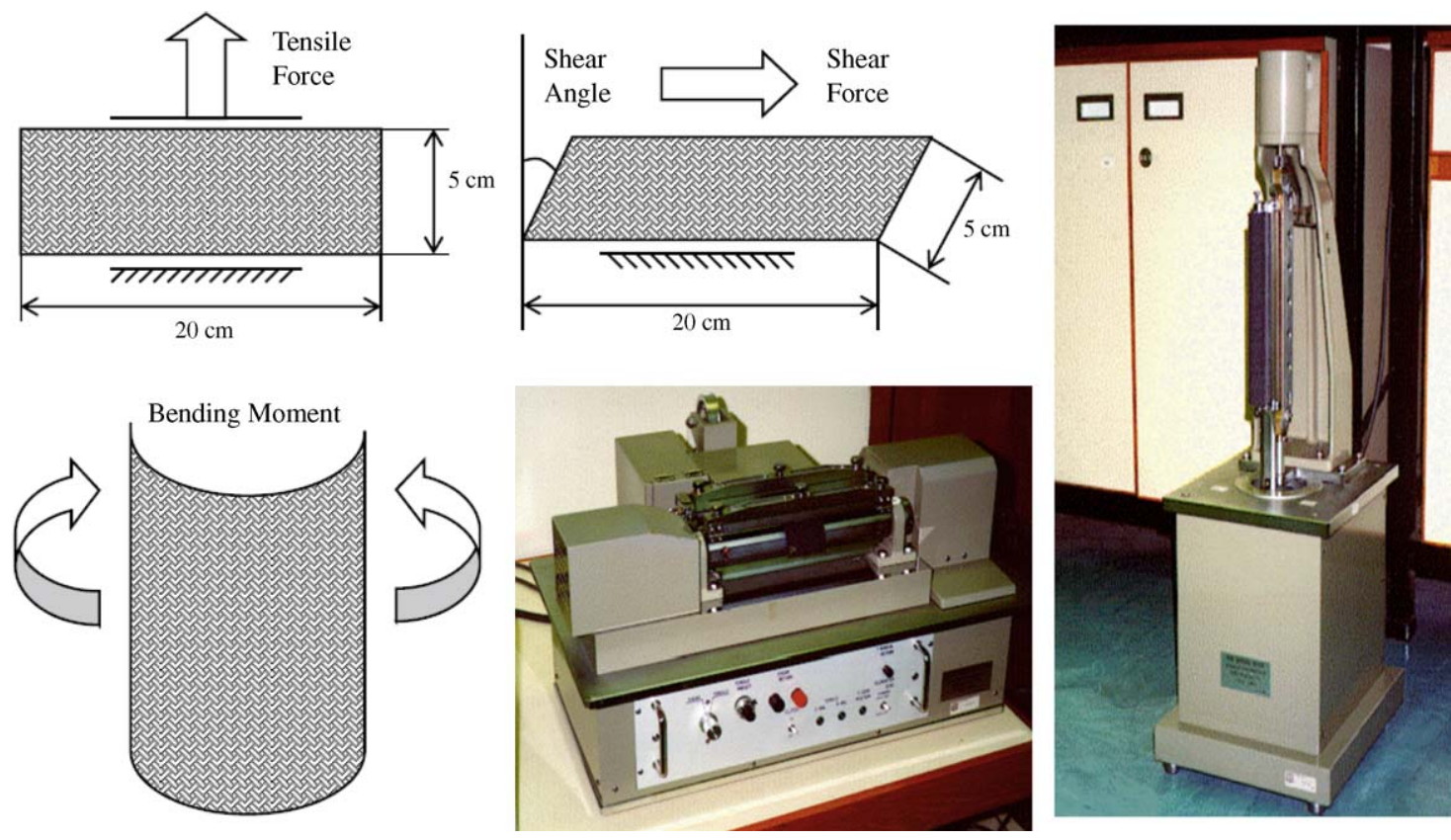

Fig. 14. The Kawabata evaluation system.

\subsection{Draping simulation and numerical schemes}

Although different energy representations $E$ are used in various deformable cloth models, there is a common basis among all the physical based simulation techniques: i.e., a system of differential equations needs to be solved numerically:

$\mathbf{M} \ddot{\mathbf{x}}=-\frac{\partial E}{\partial \mathbf{x}}+\mathbf{F}^{\prime}$

where $\mathbf{M}$ is a matrix representing mass distribution of the cloth, $\mathbf{X}$ is the position vector of particles, and $\mathbf{F}^{\prime}$ is a union of various external forces. The differential equation (2) is extremely stiff since the cloth material has strong resistance to stretch and weak resistance to bending. Let $\mathbf{F}=-(\partial E / \partial \mathbf{x})+\mathbf{F}^{\prime}$. To animate such a system, early work uses explicit numerical methods [14,36]:

$\left\{\begin{array}{l}\dot{\mathbf{x}}_{i}^{n+1}=\dot{\mathbf{x}}_{i}^{n}+\mathbf{F}_{i}^{n} \frac{\Delta t}{m} \\ \mathbf{x}_{i}^{n+1}=\mathbf{x}_{i}^{n}+\dot{\mathbf{x}}_{i}^{n+1} \Delta t\end{array}\right.$

Both Euler's methods and Runge-Kutta methods can be applied to the evolving system described in Eq. (3). Despite its ease of implementation, to ensure stability, explicit methods generally require that the time step $t$ be inverse proportional to the square root of the stiffness which is very large in cloth simulation. Thus a very small time step must be used and resist explicit methods far from real-time methods.
A novel implicit method is proposed in [9] which replaces the forces at time $t$ by the forces at time $t+\Delta t$ :

$\left\{\begin{array}{l}\dot{\mathbf{x}}_{i}^{n+1}=\dot{\mathbf{x}}_{i}^{n}+\mathbf{F}_{i}^{n+1} \frac{\Delta t}{m} \\ \mathbf{x}_{i}^{n+1}=\mathbf{x}_{i}^{n}+\dot{\mathbf{x}}_{i}^{n+1} \Delta t\end{array}\right.$

It has been observed in [9] that even for extremely stiff systems, numerical stability has not been an issue for the implicit scheme (4). To use system (4), forces $\mathbf{F}$ must be known advance at time $t+\Delta t$ : it can be predicted with a first-order approximation

$\mathbf{F}_{i}^{n+1}=\mathbf{F}_{i}^{n}+\frac{\partial \mathbf{F}}{\partial \mathbf{x}} \Delta \mathbf{x}+\frac{\partial \mathbf{F}}{\partial \dot{\mathbf{x}}} \Delta \dot{\mathbf{x}}$

Together with

$$
\begin{aligned}
\Delta \dot{\mathbf{x}} & =\mathbf{F}_{i}^{n+1} \frac{\Delta t}{m} \\
\Delta \mathbf{x} & =\left(\dot{\mathbf{x}}_{i}^{n}+\Delta \dot{\mathbf{x}}\right) \Delta t
\end{aligned}
$$

$\Delta \dot{\mathbf{x}}$ is solved by a linear system:

$$
\left[\mathbf{I}-\frac{\Delta t}{m}\left(\frac{\partial \mathbf{F}}{\partial \mathbf{x}} \Delta t+\frac{\partial \mathbf{F}}{\partial \dot{\mathbf{x}}}\right)\right] \Delta \dot{\mathbf{x}}=\frac{\Delta t}{m}\left(\mathbf{F}_{i}^{n}+\frac{\partial \mathbf{F}}{\partial \mathbf{x}} \dot{\mathbf{x}}_{i}^{n} \Delta t\right)
$$

Despite its extreme stability, the major difficulty in implicit schemes is that a large linear sparse system (5) has to be solved at each time step. Simplification can be achieved by preprocessing a linearization version of the matrix which is assumed not to change during evolution [29,50]. The goal of most implicit schemes is for real-time animation with a numerically stable solution. The results are visual pleasing for graphics display but not for engineering. For

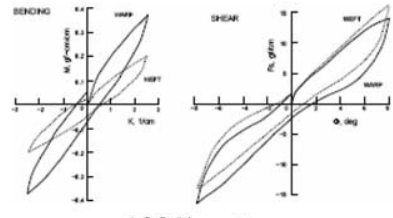

$100 \%$ cotton

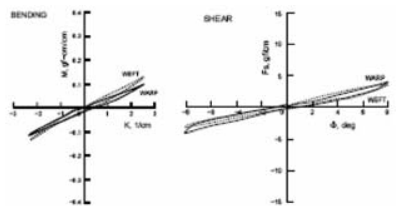

$100 \%$ wool

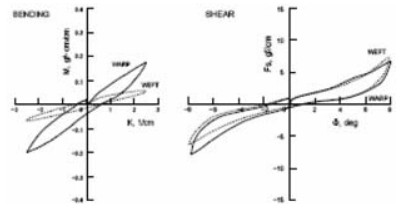

cotton-polyester

Fig. 15. Kawabata bending and shearing plots [14]. 


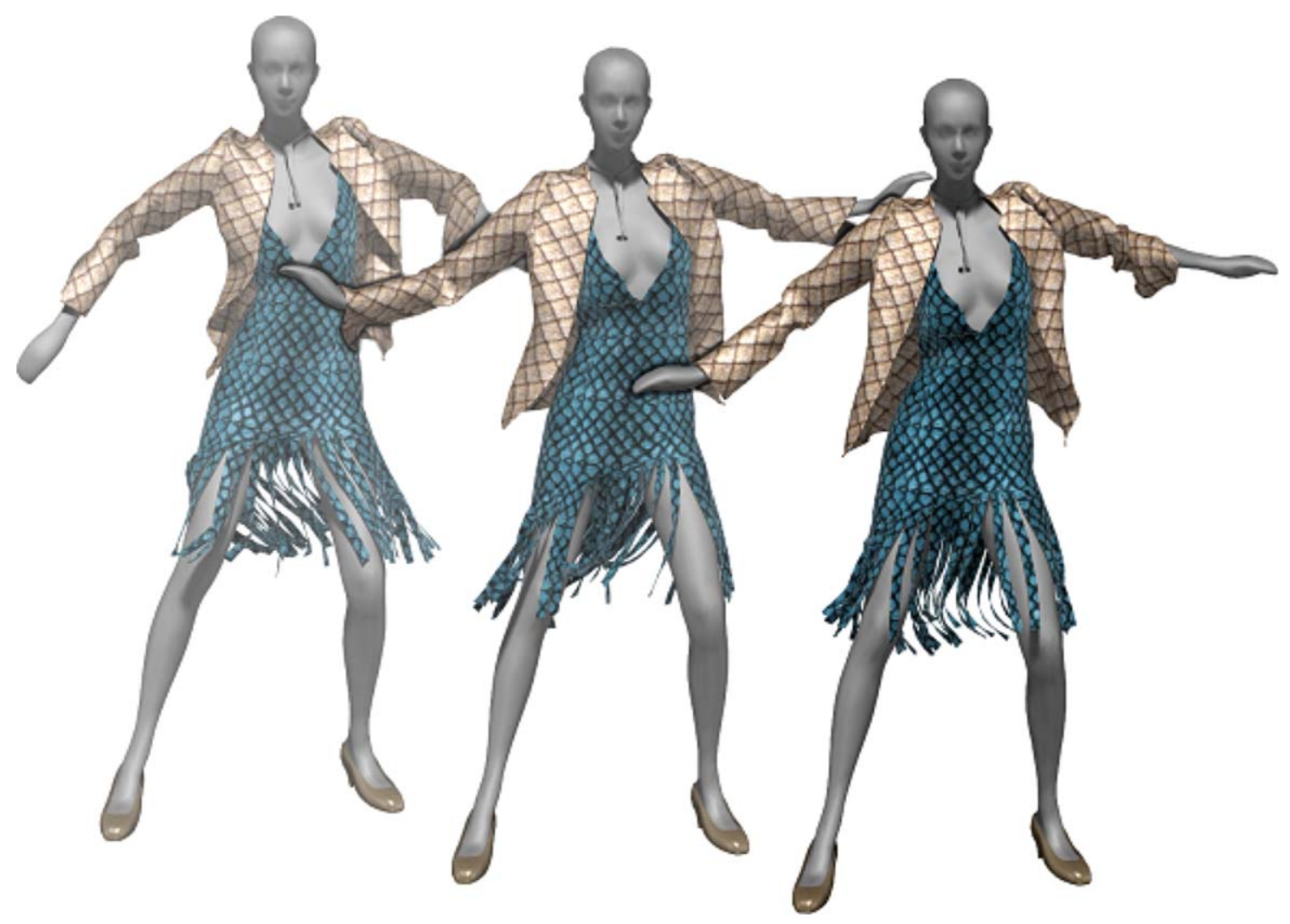

Fig. 16. Dressed human animation.

accurate simulation with moderate computational cost, the hybrid explicit/implicit algorithm in [101] is recommended.

The special property of buckling phenomena of fabrics makes it quite different from other elastic material. A deep insight is provided in [20] showing that the instability of the post-buckling response of fabrics arises from a structural instability and thus cannot be avoided by any numerical schemes. By calculating deformation energy of the shape at the predicted static equilibrium of the post-buckling state, a particle-based physical model of fabrics with a semi-implicit simulation technique is proposed in [20] which can stably integrate the equation of motion with a large fixed time step and without the need for fictitious damping forces.

Most state-of-the-art numerical schemes allow large stretches along warp directions to achieve fast performance. However, it is far from realistic since many textiles can have little stretch in physics. To solve this problem, one way is to model the cloth as a developable surface $[26,64,63]$ which cannot be stretched or torn during the animation. However, the Hamilton-principle involved numerical scheme in [64] is computationally expensive and cannot be used in real-time applications. Another promising solution [41] is based on the constrained Lagrangian mechanics, with which a novel fast projection method is developed. Experimental results show that the fast projection can produce complex, realistic simulation of cloth.

\subsection{Collision detection}

Collision detection checks whether two objects overlap in space or their boundaries share at least one common point. In computeraided design, computer graphics, robotics and computational geometry, many methods are proposed. Each of the methods takes some special properties inherent in the objects of interest and design solutions based on the application domain. An excellent review on the general collision detection is provided in [62].
In garment simulation, two types of collisions exist: selfcollisions among different parts of a single piece of clothes, and collision between the cloth model and the human model. In dressed human animation as shown in Fig. 16, detection of collision and self-collision is a bottleneck. Collision response also plays a vital role in numerical integration. Since during simulation, the configuration between geometric elements does not change very much after a small time step, the potential collision region of a geometric object must be in its close vicinity. Any efficient collision detection method should take advantage of this coherent property. Some basic coherence-based methods are studied in $[9,120]$.

To speed up the detection process, classic multiresolution techniques such as bounding volume hierarchy (BVH) methods can be used. One choice of bounding volume is to use a discrete orientation polytope (DOP) [54]. Since the shape of cloth is timedependent, to handle self-collision detection, the dynamic BVH method [118] can be applied to update the DOP-tree of the cloth model. Another speed-up technique is to use surface curvature information to pre-select the potential collision in a small region $[80,99]$.

A robust approach is proposed in [15] to handle cloth collision, contact and friction in a consistent way. The key idea is to combine a fail-safe geometric collision method with a fast (non-stiff) repulsion force method that models cloth thickness as well as both static and kinetic frictions: if the collision occurs in low velocities, penalty spring forces is applied; if in high velocities, instantaneous impulses response is executed. These two phases are switched in a fully hybridized and efficient manner.

All the above methods handle collision and contact response in a history-based manner, i.e., history information is used to help decide whether a particle penetrates inside another solid object (collision) or in the wrong side (self-collision). One shortcoming in this class of methods is that once a particle is already in wrong position, the entire simulation afterwards could be ruined. As a 
remedy, a history-free cloth collision response algorithm based on global intersection analysis of cloth meshes at each simulation step is proposed in [10].

\subsection{Limitations of existing methods}

In virtual reality and e-commerce on the internet, fast online simulation and accurate representation of cloth material behavior are both needed. However, the two goals are separated in existing methods: the material models based on Kawabata system are accurate but very slow; the spring-mass models are very fast but less accurate. Although hybrid models are proposed trying to combine the advantages of both models, there is still a long way to go in this direction. The techniques of parallel computation and GPU acceleration, surveyed in Section 8, shed some light in this challenge.

\section{3D to 2D pattern transformation}

Garment industry eventually needs 2D patterns for manufacturing. Mesh cutting operator with 3D sketch provides users a way to trim the 3D garment into piece along sewing lines. To flatten each piece from 3D space to 2D plane, two methods exist. The first method represents each piece as an elastic model with a predefined energy function. Then $3 \mathrm{D}$ to $2 \mathrm{D}$ pattern transformation corresponds to flatten the 3D model into plane with a minimal elastic deformation energy distribution. The second method first approximates each piece by a developable surface which is in turn easily developed into plane.

The first method is typified by the work in $[69,104]$. The energy function is defined similar to the one in [36]. Since only the final rest static state is needed, the motion of equations (1) can be simplified to $\mu\left(\partial^{2} x / \partial t^{2}\right)+x=0$, where the damping force is also ignored in [104]. During surface development, special attention must be paid to prevent triangles' overlap which may frequently occur. A penalty method [79] is adapted to move masses in overlapped triangles toward the opposite direction.

The surface flattening function in [104] runs in three phases. Refer to Fig. 17. Given a 3D mesh piece $M_{3 D}$, firstly an initial planar mesh $M_{2 D}^{\prime}$ with the same connectivity of $M_{3 D}$ is found by flattening triangles in $M_{3 D}$ one by one into plane. Secondly the $M_{2 D}^{\prime}$ is deformed in plane with the constraint of overlapping penalty to release the energy. When the rest state $M_{2 D}$ is reached, the energy is minimized. Thirdly, the users can optionally control the flattening accuracy by cutting the mesh. The cutting line is automatically found by seeking the most gradient descending direction in the energy field. Pseudocolor can be used to visualize the energy field.

The second method approximates each piece of 3D garment by developable surface which has a deep root in differential geometry [28]. In CAGD, most of the existing work exploits the characterizations and properties for free-form surfaces to be developable. Notably two classes of approaches exist: the first uses the primal representation of surfaces, and the second uses the dual representation of surfaces. The primal representation uses a tensor product surface of degree $(1, n)$, and usually solves nonlinear characterizing equations to guarantee developability [8,23]. A developable surface can also be viewed as the envelope of a oneparameter family of tangent planes, and thus can be treated as a curve in a dual projective 3-space. Given this dual representation, some interpolation and approximation algorithms can be computed efficiently $[12,78]$.

A novel technique for developable approximation of garment pieces is proposed in [26]. Three steps are involved. Firstly for each triangle in the original pieces, a smooth developable surface is found to best approximate its neighboring area. Then in the second step, each triangle is moved into its approximated surface with minimal change of vertex position. All adjusted triangles are glued together by preserving the newly computed normals and positions. Finally the resulted meshes are unfolded into plane with minimal shearing by using the $\mathrm{ABF}++$ conformal parameterization [86].

\subsection{Limitations of existing methods}

Most existing methods for pattern flattening have not taken material properties into account. Motivated by the mathematic properties of developable surfaces [28], the flattening of free-form surfaces usually minimizes some measures of distortion character-
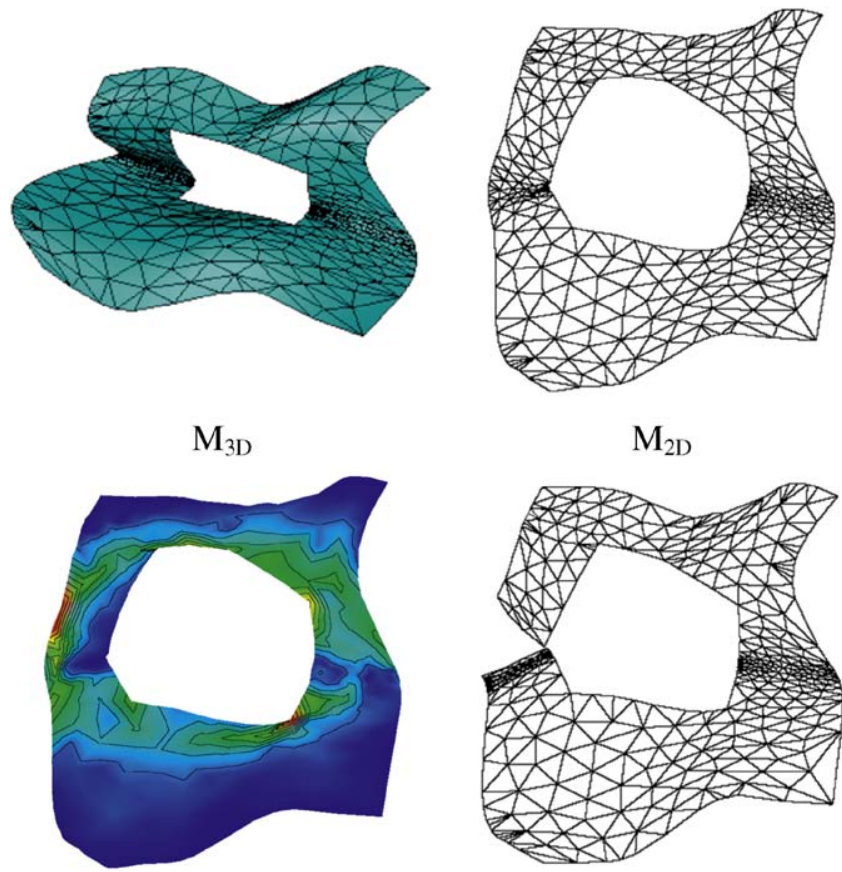

Isolines in the energy field

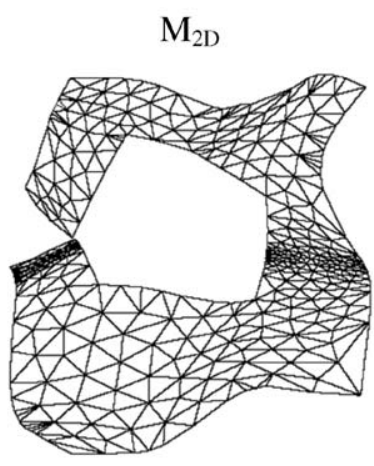

Cutting along the gradient

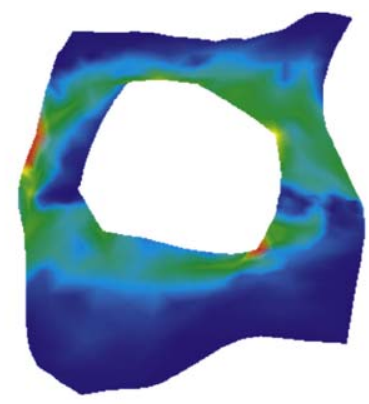

Energy field of $\mathrm{M}_{2 \mathrm{D}}$

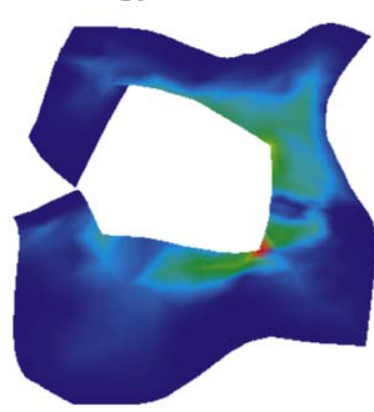

Energy field after cutting

Fig. 17. Surface flattening by energy release [104] (courtesy of Prof. Charlie C.L. Wang). 
ized by the first fundamental form of surface. These measures are geometry-oriented such that fast and stable numerical process can be achieved. It is interesting to ask, can the measures be materialoriented? Given a piece of 3D cloth with the same geometry but different material, can we get different $2 \mathrm{D}$ patterns by flattening? It is expected that the future work can ask these questions.

\section{Geometric detail modeling}

Numerical animation of deformable cloth models is a way to generate realistic fine details on garment draping in real time. In computer-aided design, garment draping results of dressed human can be pre-computed offline. Therefore transferring fine details in pre-computed garment from the standard-size human to a customized human is another useful technique which can quickly visualize the accurate fitting effect of garment style on customized bodies.

Shell map [77] is proposed as a bijective mapping between shell space and texture space that can generate small-scale features on surfaces using a variety of modeling techniques. In terms of modeling fine details on garment surface $M$, the isosurfaces in the shell $\left\{x \in \mathbb{R}^{3},|x-p| \leq \varepsilon, p \in M\right\}$ are constructed, where $2 \varepsilon$ is the shell thickness. Refer to Fig. 18. The fine details are encoded in the space between the base garment surface and its offsets characterized by isosurfaces. Given the base garment surfaces designed from the feature template as shown in Fig. 9, the same garment template can be used in any customized human body with different sizes. By decoding the details with the same scalar coefficients in the isosurfaces around the new base garment surface, fine details are naturally grown on the new customized garments.

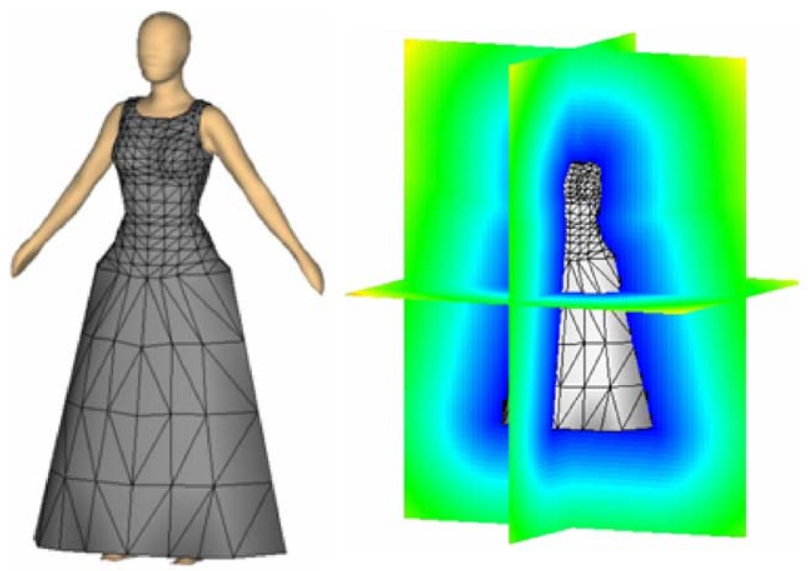

(a) isosurfaces in the shell around the base cloth surface

(c1)

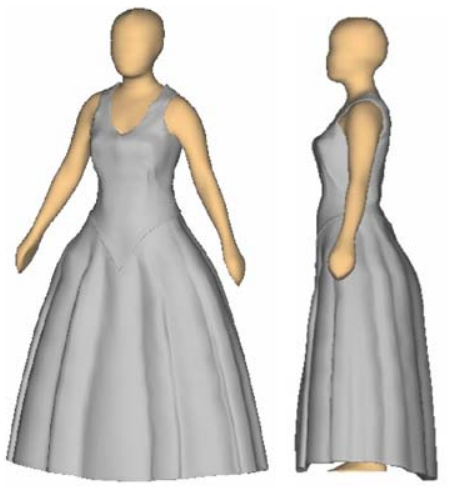

(c2)

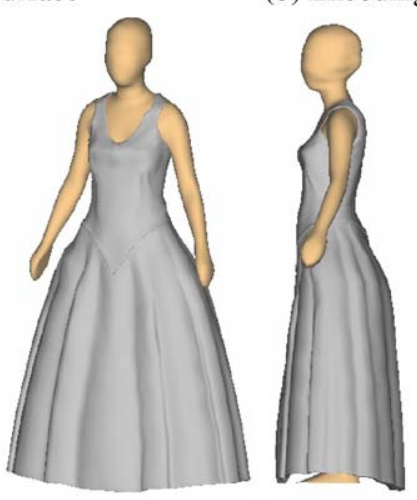

(c3)
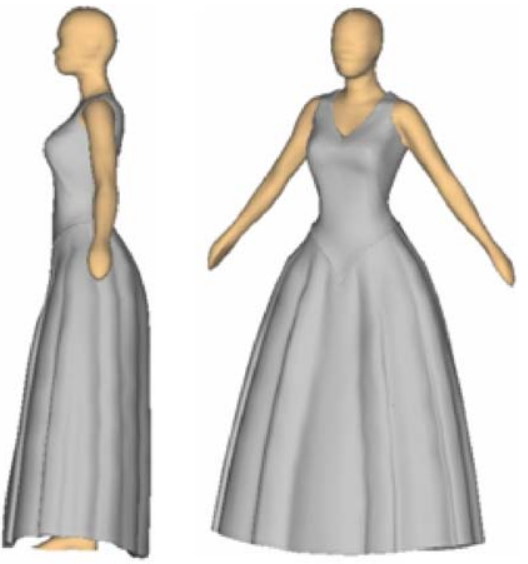

Shell map can be regarded as one instantiation of volume parameterization [103]. The purpose of volume parameterization is to establish a mapping between the spaces that are near to two reference free-form models, so that the shape of a product presented in free-form surfaces can be transferred from the space around one reference model to another reference model. The mapping is expected to keep the spatial relationship between the product model and reference models as much as possible (ref. Fig. 19). In [61] a harmonic volumetric mapping is proposed to establish a smooth bijective correspondence between two solid shapes with the same topology. More applications such as information transfer, shape registration, deformation sequence analysis and solid texture analysis are demonstrated, showing the power of volume parameterization in fine details encoding. Note that non-manifold structures are frequently appeared in garment such as collars, epaulets, sleeves and so on. Volume parameterization is versatile in handling geometric details, regardless of manifoldness or non-manifoldness of the object's surfaces (ref. Fig. 20).

\section{Parallel computation and GPU acceleration}

Garment simulation with appearance of accurate fine details, such as folding and wrinkles, is computationally intensive. Parallel computing is promising for data-intensive scientific computing [56]. Two types of parallel computation exist: computation on graphics hardware and computation on multi-core processor architectures. The numerical integration of garment simulation including solving large sparse linear systems in implicit schemes is highly parallelizable. Physically-based simulation with parallel

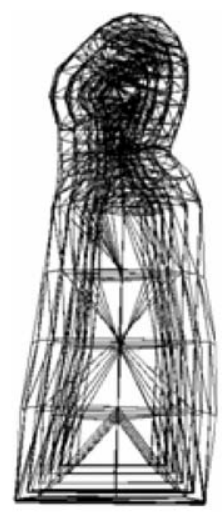

(b) Encoding fine details in isosurface

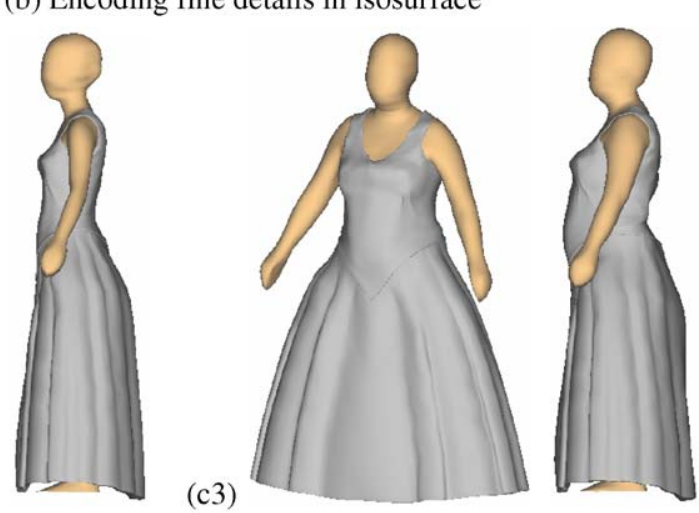

(c) Fine details transformation between customized garments with different sizes

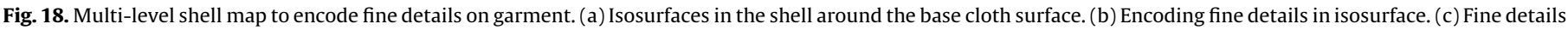
transformation between customized garments with different sizes. 

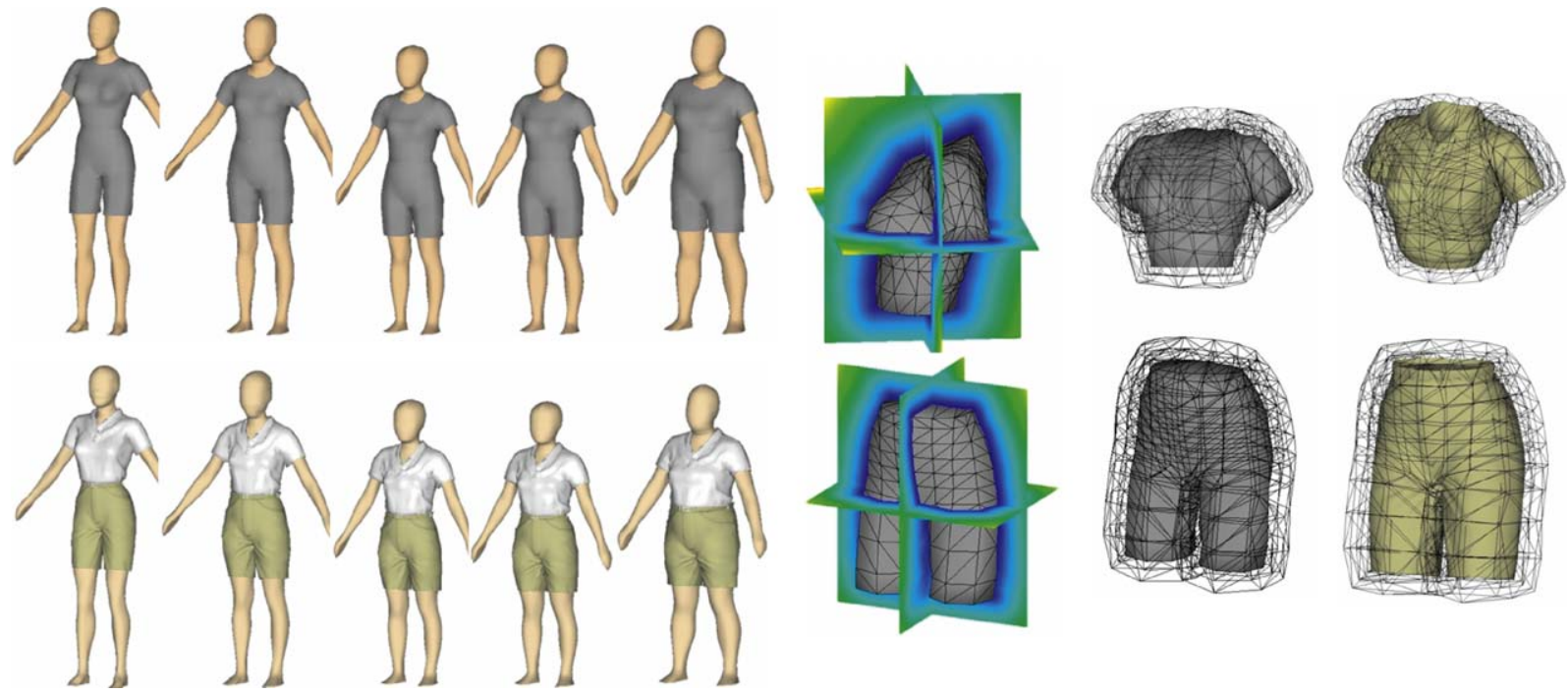

Fig. 19. Fine details auto-generation on polo shirt and short pant.

conjugate gradients methods and parallel collision detection techniques, on multi-core processors, is studied in [94]. Stateof-the-art parallel techniques also model and transfer the fine details on cloth surfaces in parallel.

Commodity computer graphics chips, known as Graphics Processing Units (GPUs), can provide tremendous memory bandwidth and computational horsepower. In [66] a GPU-based method is proposed to generate and render detailed folds on garment. The core of this parallel technique is to determine in a controllable way a highly detailed, globally consistent wrinkle field from arbitrary geometric deformations of a cloth mesh. For this purpose, the cloth mesh is arranged into a format in which the adjacency data for the unified vertices are stored in a buffer of size $512 \times 512$. Given this structure, the cloth deformation is performed by driving a skeleton to which the mesh is attached by soft skinning: this is done by a local linear approximation in the neighborhood of each vertex and can be handled in vertex shader. Then the cloth model is crushed by computing the compression ratio using the arc length of sinusoidal wrinkles. The wrinkled field is post-processed by relaxation that aligns the waves of

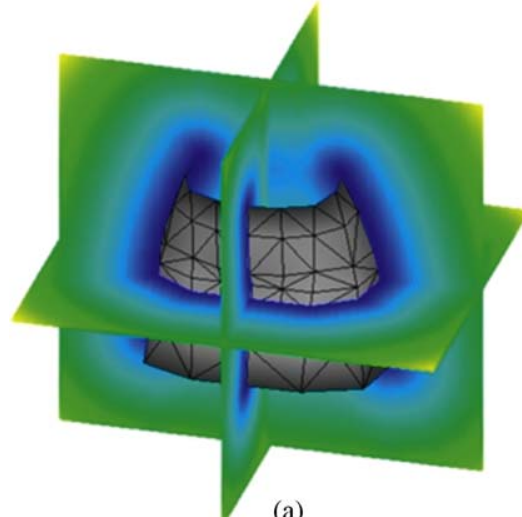

(a)

Distance field for base surface

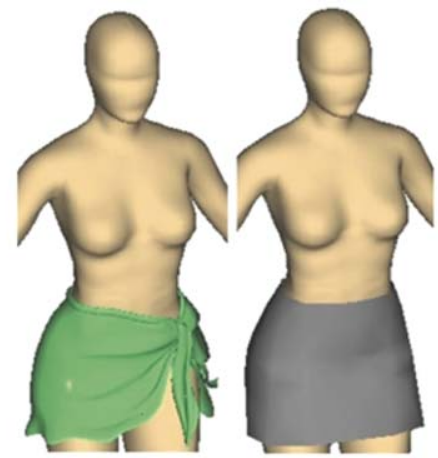

(d) coarse and refined skirt on model A

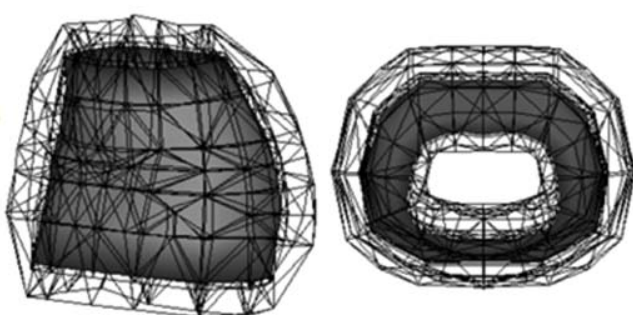

(b)

shells shown in two views

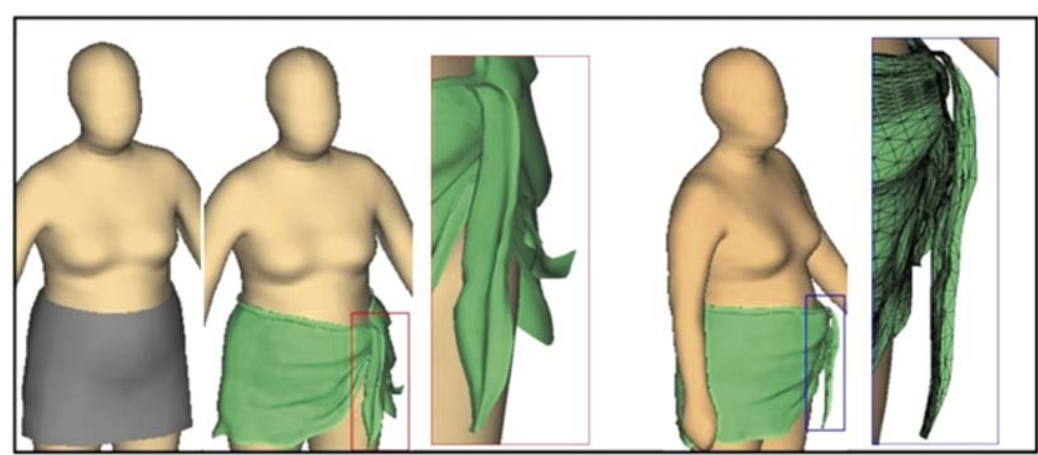

(e) Fringe reconstruction to model $\mathbf{E}$

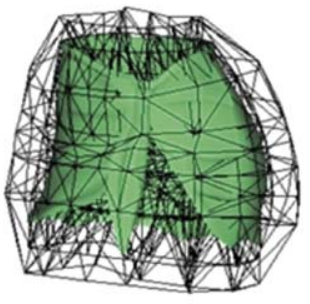

(c)

insert refined skirt to shell space

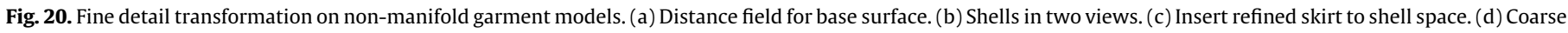
and refined skirt on model A. (e) Fringe reconstruction to model E. 
neighboring vertices. In all the phases of skin, crush and relax, all vertices are mapped to single pixels in two off-screen floatingpoint buffers of size $256 \times 256$ : one for position data and one for normal data. Finally the wrinkled cloth mesh is rendering with texture by vertex and pixel shaders.

Many authors have proposed diverse GPU-based methods for modeling geometry of general fine details, which are mostly characterized by the normal field [68] and the displacement map [91]. A good survey on general-purpose computing on the GPU can be found in [73]. [34] gives a good summary on GPU cluster for high performance computing. The authors further present a general framework for computation and visualization on a GPU clusters [35]. It is expected that more and more computational loads in garment simulation will be shifted into GPUs and distributed in parallel into multi-core processors.

\section{Future directions}

Despite significant progresses have been made over the last decade, several potential avenues exist toward innovative design techniques in future 3D garment CAD systems. Three challenging research problems in physically-based simulation of cloth models are depicted in [21]. Below we propose two research directions for future garment CAD development.

Artificial Intelligence and machine learning in garment design. Garment design and simulation involves intensive data creation and transformation between different modeling stages. Although fast numerical schemes and parallel techniques are proposed, clever synthesis and reuse of existing data can be beneficial. Machine learning [70] is a system of techniques that allows users to leverage existing data in a non-direct and non-trivial manner. Expert pattern systems based on artificial intelligence (AI) have already been widely used in 2D garment CAD systems to reduce the experience requirement. A fuzzy logic method is also used to construct 3D individuals' digital body from laser scanners [107]. It is expected that:

- More AI techniques should involve in 3D garment design for data creation and analysis, such as determining sizing tables and material properties by automatically recognizing sketches of fashion designer;

- Machine learning techniques should be emphasized to reuse existing data such as wrinkle styles of particular cloth materials to auto-generate/synthesize new data on new designed garment with customer sizes.

Real-time collaborative environment of garment product development. Nowadays garment product development activities are segmented and operated in various geographically dispersed cities and countries, exhibiting strong needs for collaborative design and manufacturing. Massive design data, including text, 2D sketch and 3D CAD model data, are stored at various means such as paper, bitmap image files, CAD model files, $C D$, diskette, FTP storage, email, fax and verbal. To enable users to develop, view, manipulate, analyze, and integrate product designs in a collaborative environment for multi-national manufacturing companies, it is promising in the next generation to use a real-time collaborative product development environment for 3D CAD data sharing and manipulation, on the basis of supporting interactive collaborative design and manufacturing activities.

\section{Conclusions}

In this paper we present a comprehensive review on CAD methods in 3D garment design in the field of fashion design and manufacture engineering. Several key modules in 3D garment design are coherently summarized. First the basic design methodology is outlined. Then feature modeling is presented as the core of 3D garment design technology platforms. Towards fast and accurate garment simulation, numerical integration and parallel computing techniques are depicted as the numerical engine in the design platform. CAD methods on modeling fine geometric details are also included to add realism to the garment exhibition.

3D garment design remains an active area of research. This survey is not meant to be complete. We also did not address the important applications of garment design in computer animation. Nevertheless, we hope that this paper can provide some help for researchers to review the past developments and identify possible directions for future research on 3D CAD methods in garment design.

\section{Acknowledgements}

The authors thank the guest editors and reviewers for their valuable comments. This work was partially supported by the National Natural Science Foundation of China (Project Number 60603085, 60736019, 60970099) and the National High Technology Research and Development Program of China (Project Number 2007AA01Z336).

\section{References}

[1] B. Allen, B. Curless, Z. Popovic, The space of human body shapes: reconstruction and parameterization from range scans, in: ACM SIGGRAPH 2003, 2003, 587594.

[2] M. Alexa, J. Behr, D. Cohen-Or, S. Fleishman, D. Levin, C.T. Silva, Computing and rendering point set surfaces, IEEE Transactions on Visualization and Computer Graphics 9 (1) (2003) 3-15.

[3] N. Amenta, M. Bern, M. Kamvysselis, A new Voronoi-based surface reconstruction algorithm, in: ACM SIGGRAPH 1998, 1998, 415-421.

[4] M. Aono, A wrinkle propagation model for cloth, in: Proc. Computer Graphics International'96, 1996, 95-115.

[5] M. Aono, D.E. Breen, M.J. Wozny, Fitting a woven-cloth to a curved surface: mapping algorithms, Computer-Aided Design 26 (4) (1994) 278-292.

[6] C.K. Au, M.M.F. Yuen, Feature-based reverse engineering of mannequin for garment design, Computer-Aided Design 31 (12) (1999) 751-759.

[7] C.K. Au, M.M.F. Yuen, A semantic feature language for sculptured object modeling, Computer-Aided Design 32 (1) (2000) 63-74.

[8] G. Aumann, A simple algorithm for designing developable Bezier surfaces, Computer Aided Geometric Design 20 (2003) 601-619.

[9] D. Baraff, A. Witkin, Large steps in cloth simulation, in: ACM SIGGRAPH 1998, 1998, 43-54.

[10] D. Baraff, A. Witkin, M. Kass, Untangling cloth, in: ACM SIGGRAPH 2003, 2003, 862-870.

[11] J. Bloomenthal, C. Bajaj, J. Blinn, M. Cani-Gascuel, A. Rockwood, B. Wyvill, G. Wyvill, Introduction to Implicit Surfaces, Morgan Kaufmann Publishers, Inc., 1997.

[12] R. Bodduluri, B. Ravani, Design of developable surfaces using duality between plane and point geometries, Computer-Aided Design 25 (1993) 621-632.

[13] D.E. Breen, D.H. House, P.H. Getto, A physically-based particle model of woven cloth, Visual Computer 8 (5-6) (1992) 264-277.

[14] D.E. Breen, D.H. House, M.J. Wozny, Predicting the drape of woven cloth using interacting particles, in: ACM SIGGRAPH 1994, pp. 365-372.

[15] R. Bridson, R. Fedkiw, J. Anderson, Robust treatment of collisions, contact, and friction for cloth animation, in: ACM SIGGRAPH 2002, 2002, 594-603.

[16] K. Case, J. Gao, Feature technology: an overview, International Journal of Computer Integrated Manufacturing 6 (1-2) (1993) 2-12.

[17] M. Carignan, Y. Yang, N. Magnenat-Thalmann, D. Thalmann, Dressing animated synthetic actors with complex deformable clothes, in: ACM SIGGRAPH 1992, 1992, 99-104.

[18] J.C. Carr, R.K. Betson, J.B. Cherrie, T.J. Mitchell, W.R. Fright, B.C. McCallum, T.R. Evans, Reconstruction and representation of 3D objects with radial basis functions, in: ACM SIGGRAPH 2001, 2001, 67-76.

[19] S.W. Cheng, T.K. Dey, E. Ramos, T. Ray, Sampling and meshing a surface with guaranteed topology and geometry, SIAM Journal Computing 37 (2007) 11991227.

[20] K.J. Choi, H.S. Ko, Stable but responsive cloth, in: ACM SIGGRAPH 2002, 2002.

[21] K.J. Choi, H.S. Ko, Research problems in cloth simulation, Computer-Aided Design 37 (6) (2005) 585-592.

[22] K.J. Choi, H.S. Ko, R. Fedkiw, D.L. Zhang, Advanced Topics on Clothing Simulation and Animation, in: ACM SIGGRAPH 2005 Course Notes 6, Los Angeles, USA.

[23] C. Chu, C.H. Sequin, Developable Bezier patches: properties and design, Computer-Aided Design 34 (2002) 511-527. 
[24] F. Cordier, N. Magnenat-Thalmann, Real-time animation of dressed virtual humans, Eurographics (2002) 327-336.

[25] B. Curless, M. Levoy, A volumetric method for building complex models from range images, in: SIGGRAPH 1996, 1996, 303-312.

[26] P. Decaudin, D. Julius, J. Wither, L. Boissieux, A. Sheffer, M.P. Cani, Virtual garments: a fully geometric approach for clothing design, Eurographics (2006) 625-634.

[27] E. den Berg, W.F. Bronsvoort, J.S.M. Vergeest, Freeform feature modelling: concepts and prospects, Computers in Industry 49 (2) (2002) 217-233.

[28] M.P. do Carmo, Differential Geometry of Curves and Surfaces, Prentice-Hall, Englewood Cliffs, NJ, 1976.

[29] M. Desbrun, P. Schroder, A.H. Barr, Interactive animation of structured deformable objects, Graphics Interface (1999) 1-8.

[30] N. Dyn, D. Levin, J.A. Gregory, A butterfly subdivision scheme for surface interpolation with tension control, ACM Transactions on Graphics 9 (2) (1990) 160-169.

[31] B. Eberhardt, A. Weber, W. Strasser, A fast, flexible, particle-system model for cloth draping, IEEE Computer Graphics and Applications (1996) 52-59.

[32] M. Eck, H. Hoppe, Automatic reconstruction of B-spline surfaces of arbitrary topological type, in: SIGGRAPH 1996, 1996, 325-334.

[33] J.W. Eischen, S. Deng, T.G. Clapp, Finite-element modeling and control of flexible fabric parts, IEEE Computer Graphics and Applications (1996) 71-80.

[34] Z. Fan, F. Qiu, A. Kaufman, S. Yoakum-Stover, GPU cluster for high performance computing, in: ACM/IEEE Supercomputing Conference 2004, 2004, 47-58.

[35] Z. Fan, F. Qiu, A. Kaufman, Zippy: a framework for computation and visualization on a GPU cluster, Computer Graphics Forum 27 (2) (2008) 341-350.

[36] J. Fan, Q. Wang, S.F. Chen, M.M.F. Yuen, C.C. Chan, A spring-mass model-based approach for warping cloth patterns on 3D objects, Journal of Visualization and Computer Animation 9 (4) (1998) 215-227.

[37] G. Farin, Curves and Surfaces for CAGD: A Practical Guide, 5th ed., Morgan Kaufmann, San Francisco, CA, 2002.

[38] P.A. Fitzhorn, Formal graph languages of shape, Artificial Intelligence for Engineering, Design and Manufacturing 4 (3) (1990) 151-164.

[39] J. Fu, A. Joneja, K. Tang, Modeling wrinkles on smooth surfaces for footwear design, Computer-Aided Design 37 (8) (2005) 815-823.

[40] T. Funkhouser, M. Kazhdan, P. Shilane, P. Min, M. Kiefer, A. Tal, S. Rusinkiewicz, D. Dobkin, Modeling by example, in: ACM SIGGRAPH 2004, 2004, 652-663.

[41] R. Goldenthal, D. Harmon, R. Fattal, M. Bercovier, E. Grinspun, Efficient simulation of inextensible cloth, in: ACM SIGGRAPH 2007, 2007.

[42] J.A. Gregory, N-sided surface patches, in: The Mathematics of Surfaces, Oxford University Press, Oxford, 1986, pp. 217-232.

[43] I. Guskov, K. Vidimce, W. Sweldens, P. Schröder, Normal meshes, in: ACM SIGGRAPH 2000, 2000, 95-102.

[44] S. Hadap, E. Bongarter, P. Volino, N. Magnenat-Thalmann, Animating wrinkles on clothes, in: Proceedings of IEEE Visualization '99, 1999, pp. 175-182.

[45] B.K. Hinds, J. McCartney, Interactive garment design, Visual Computer 6 (2) (1990) 53-61.

[46] H. Hoppe, T. DeRose, T. Duchamp, J. McDonald, W. Stuetzle, Surface reconstruction from unorganized points, in: ACM SIGGRAPH 1992, 1992, 71-78.

[47] D.H. House, D.E. Breen, Cloth Modeling and Animation, A. K. Peters, 2000.

[48] T. Igarashi, T. Moscovich, J.F. Hughes, As-rigid-as-possible shape manipulation, in: ACM SIGGRAPH 2005, 2005.

[49] T. Igarashi, S. Matsuoka, H. Tanaka, Teddy: a sketching interface for 3D freeform design, in: ACM SIGGRAPH 1999, 1999, pp. 409-416.

[50] Y.M. Kang, J.H. Choi, H.G. Cho, D.H. Lee, An efficient animation of wrinkled cloth with approximate implicit integration, Visual Computer 17 (3) (2001) 147157.

[51] S. Kawabata, The Standardization and Analysis of Hand Evaluation, The Textile Machinery Society of Japan, Osaka, 1980.

[52] S. Kim, T. Kang, Garment pattern generation from body scan data, ComputerAided Design 35 (7) (2003) 611-618.

[53] S. Kim, C.K. Park, Basic garment pattern generation using geometric modeling method, International Journal of Clothing Science and Technology 19 (1) (2007) 7-17.

[54] J. Klosowski, T. Held, M. Mitchell JSB, H. Sowizral, K. Zikan, Efficient collision detection using bounding volume hierarchies of k-DOPs, IEEE Transactions on Visualization and Computer Graphics 4 (1) (1998) 21-36.

[55] L.P. Kobbelt, Discrete fairing and variational subdivision for free form surface design, Visual Computer 16 (3-4) (2000) 142-158.

[56] V. Kumar, A.A. Gupta, An Introduction to Parallel Computing: Design and Analysis of Algorithms, 2nd ed., Benjamin/Cummings Publishing Company, 1994.

[57] T.L. Kunii, H. Gotoda, Singularity theoretical modeling and animation of garment wrinkle formation processes, Visual Computer 6 (6) (1990) 326-336.

[58] W. Lee, J. Gu, N. Magnenat-Thalmann, Generating animatable 3D virtual humans from photographs, in: EUROGRAPHICS 2000, 2000, 1-10.

[59] A. Lee, H. Moreton, H. Hoppe, Displaced subdivision surfaces, in: ACM SIGGRAPH $2000,2000,85-94$.

[60] C.L. Li, K.C. Hui, Feature recognition by template matching, Computers and Graphics 24 (4) (2000) 569-582.

[61] X. Li, X. Guo, H. Wang, Y. He, X. Gu, H. Qin, Harmonic volumetric mapping for solid modeling applications, in: Proc. of ACM Solid and Physical Modeling Symposium, 2007, 109-120.

[62] M.C. Lin, D. Manocha, Collision and proximity queries, Handbook of Discrete and Computational Geometry: Collision Detection, 2003.
[63] Y.J. Liu, Y.K. Lai, S.M. Hu, Stripification of free-form surfaces with global error bounds for developable approximation. IEEE Transactions on Automation Science and Engineering 6 (4) (2009) 700-709.

[64] Y.J. Liu, K. Tang, A. Joneja, Modeling dynamic developable meshes by the Hamilton principle, Computer-Aided Design 39 (9) (2007) 719-731.

[65] Y.J. Liu, M.M.F. Yuen, S. Xiong, A feature-based approach for individualized human head modeling, Visual Computer 18 (5-6) (2002) 368-381.

[66] J. Loviscach, Wrinkling coarse meshes on the GPU, Eurographics (2006) 467-476.

[67] N. Magnenat-Thalmann, D. Thalmann, Virtual humans: thirty years of research, what next? Visual Computer 21 (12) (2005) 997-1015.

[68] M. Marinov, M. Botsch, L. Kobbelt, GPU-based multiresolution deformation using approximate normal field reconstruction, ACM Journal of Graphics Tools 12 (1) (2007) 27-46.

[69] J. McCartney, B.K. Hinds, B.L. Seow, The flattening of triangulated surfaces incorporating darts and gussets, Computer-Aided Design 31 (4) (1999) 249-260.

[70] T. Mitchell, Machine Learning, WCB/McGraw-Hill, 1997.

[71] N.J. Mitra, L.J. Guibas, M. Pauly, Symmetrization, in: ACM SIGGRAPH 2007, 2007, Article 63.

[72] H. Okabe, H. Imaoka, et al., Three dimensional apparel CAD system, Computer Graphics 26 (2) (1992) 105-110.

[73] J.D. Owens, D. Luebke, N. Govindaraju, M. Harris, J. Kruger, A. Lefohn, T. Purcell, A survey of general-purpose computation on graphics hardware, Computer Graphics Forum 26 (1) (2007) 80-113.

[74] S. Petrak, D. Rogale, Systematic representation and application of a 3D computeraided garment construction method. Part I, International Journal of Clothing Science and Technology 18 (3) (2006) 179-187.

[75] L. Piegl, W. Tiller, The NURBS Book, 2nd ed., Springer, Berlin, New York, 1997.

[76] I. Pitas, Digital Image Processing: Algorithms and Applications, Wiley, New York, 2000.

[77] S.D. Porumbescu, B. Budge, L. Feng, K. Joy, Shell maps, in: ACM SIGGRAPH 2005 , 2005, 626-633.

[78] H. Pottmann, G. Farin, Developable rational Bezier and B-spline surfaces, Computer Aided Geometric Design 12 (1995) 513-531.

[79] X. Provot, Deformation constraint in a mass-spring model to describe rigid cloth behavior, in: Proceedings of Graphics Interface'95, 1995, pp. 147-154.

[80] X. Provot, Collision and self-collision handling in cloth model dedicated to design garments, in: Proceedings of Graphics Interface'97, 1997, pp. 177-189.

[81] P. Rademacher, View-dependent geometry, in: ACM SIGGRAPH 1999, 1999, 439446.

[82] P. Ratner, 3-D Human Modeling and Animation, 2nd ed., Wiley, New York, 2003.

[83] T.W. Sederberg, D.L. Cardon, G.T. Finnigan, N.S. North, J. Zheng, T. Lyche, T-spline simplification and local refinement, in: ACM SIGGRAPH 2004, 2004.

[84] H. Seo, N. Magnenat-Thalmann, An automatic modeling of human bodies from sizing parameters, in: ACM SIGGRAPH 2003 Symposium on Interactive 3D Graphics, 2003, 19-26.

[85] H. Seo, N. Magnenat-Thalmann, An example-based approach to human body manipulation, Graphical Models 66 (1) (2004) 1-23.

[86] A. Sheffer, B. Levy, M. Mogilnitsky, A. Bogomyakov, ABF++: fast and robust angle based flattening, ACM Transactions on Graphics 24 (2) (2005) 311-330.

[87] P.P.J. Sloan, C.F. Rose, M.F. Cohen III, Shape by example, in: Proceedings of the 2001 Symposium on Interactive 3D graphics 2001, 2001, pp. 135-143.

[88] J. Solinger, Apparel Manufacturing Handbook: Analysis, Principles, and Practice, Bobbin Media Corp, Columbia, SC, 1988.

[89] I.H. Sul, T.J. Kang, Interactive garment pattern design using virtual scissoring method, International Journal of Clothing Science and Technology 18 (1) (2006) 31-42.

[90] H. Suzuki, Y. Sakurai, T. Kanai, F. Kimura, Interactive mesh dragging with an adaptive remeshing technique, Visual Computer 16 (3-4) (2000) 159-176.

[91] L. Szirmay-Kalos, T. Umenhoffer, Displacement mapping on the GPU-state of the art, Computer Graphics Forum 27 (6) (2008) 1567-1592.

[92] P.J. Taylor, M.M. Shoben, Grading for the Fashion Industry: The Theory and Practice, England Thornes, Cheltenham, 1990.

[93] D. Terzopoulos, J.C. Platt, H. Barr, Elastically deformable models, in: ACM SIGGRAPH 1987, pp. 205-214.

[94] B. Thomaszewski, S. Pabst, W. Blochinger, Parallel techniques for physicallybased simulation on multi-core process architectures, Computers \& Graphics 32 (1) (2008) 25-40.

[95] E. Turquin, M.P. Cani, J.F. Hughes, Sketching garments for virtual characters, in: EG Workshop on Sketch-Based Interfaces and Modeling 2004, 2004, 175-182.

[96] P. Volino, F. Cordier, N. Magnenat-Thalmann, Versatile and efficient technique for simulating cloth and other deformation objects, in: ACM SIGGRAPH 2005 1995, 137-144.

[97] P. Volino, F. Cordier, N. Magnenat-Thalmann, From early virtual garment simulation to interactive fashion design, Computer-Aided Design 37 (6) (2005) 593608.

[98] L. Velho, J. Gomes, L. Henrique, Implicit Object in Computer Graphics, SpringerVerlag, New York, Hong Kong, 2002.

[99] P. Volino, N. Magnenat-Thalmann, Collision and self-collision detection: efficient and robust solutions for highly deformable surfaces, in: Sixth Eurographics Workshop on Animation and Simulation, 1995, 55-65.

[100] P. Volino, N. Magnenat-Thalmann, Virtual Clothing: Theory and Practice, Springer, 2000

[101] P. Volino, N. Magnenat-Thalmann, Implementing fast cloth simulation with collision response, in Computer Graphics International'00, 2000, pp. 257-266.

[102] C.C.L. Wang, Parameterization and parametric design of mannequins, ComputerAided Design 37 (1) (2005) 83-98. 
[103] C.C.L. Wang, K.C. Hui, K.M. Tong, Volume parameterization for design automation of customized free-form products, IEEE Transactions on Automation Science and Engineering 4 (1) (2007) 11-21.

[104] C.C.L. Wang, S.S.F. Smith, M.M..F. Yuen, Surface flattening based on energy model, Computer-Aided Design 34 (11) (2002) 823-833.

[105] C.C.L. Wang, Y. Wang, M.M.F. Yuen, Feature based 3D garment design through 2D sketches, Computer-Aided Design 35 (7) (2003) 659-672.

[106] C.C.L. Wang, Y. Wang, M.M.F. Yuen, Design automation for customized apparel products, Computer-Aided Design 37 (7) (2005) 675-691.

[107] C.C.L. Wang, K.K. Chang, M.M.F. Yuen, From laser-scanned data to feature human model: a system based on fuzzy logic concept, Computer-Aided Design 35 (3) (2003) 241-253.

[108] C.C.L. Wang, Y. Wang, K.K. Chang, M.M.F. Yuen, Virtual human model from photographs for garment industry, Computer-Aided Design 35 (6) (2003) 577-589.

[109] J. Weil, The synthesis of cloth objects, in: Computer Graphics Proceedings'86, 1990, pp. 49-53.

[110] V. Weiss, L. Andor, G. Renner, T. Varady, Advanced surface fitting techniques, Computer Aided Geometric Design 19 (1) (2002) 19-42.

[111] http://www.toray-acs.jp/english/.

[112] http://www.gerbertechnology.com/.

[113] http://www.investronica-sis.es/.

[114] http://www.lectra.com/en/index.html.

[115] http://www.assyst-intl.com/.

[116] http://www.dressingsim.com/.

[117] Y. Yang, W. Zhang, Investigating the development of digital patterns for customized apparel, International Journal of Clothing Science and Technology 19 (34) (2007) 167-177.

[118] G. Zachmann, Rapid collision detection by dynamically aligned DOP-tree, in: Proc. IEEE Virtual Reality Annual International Symposium'98, 1998, 90-97.

[119] R.C. Zeleznik, K.P. Herndon, J.F. Hughes, SKETCH: an interface for sketching 3D scenes, in: ACM SIGGRAPH 1996, 1996, 163-170.

[120] D.L. Zhang, M.M.F. Yuen, A coherence-based collision detection method for dressed human simulation, Computer Graphics Forum 12 (1) (2002) 33-42.

[121] H.K. Zhao, S. Osher, B. Merriman, M. Kang, Implicit and nonparametric shape reconstruction from unorganized data using a variational level set method, Computer Vision and Image Understanding 80 (3) (2000) 295-314.

[122] Y. Zhong, B. Xu, Automatic segmenting and measurement on scanned human body, International Journal of Clothing Science and Technology 18 (1) (2006) 19-30.

[123] D. Zorin, P. Schroder, Subdivsion for modeling and animation, in: SIGGRAPH Course Notes, 2000.

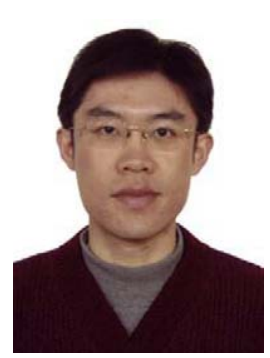

Yong-Jin Liu received his Ph.D. from the Hong Kong University of Science and Technology, Hong Kong, in 2003. He is an Associate Professor with the Department of Computer Science and Technology, Tsinghua University, Beijing, China. His research interests include computer graphics and computer-aided design.

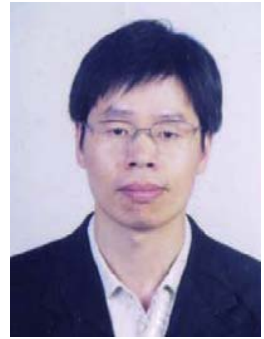

Dong-Liang Zhang received his Ph.D. from the Hong Kong University of Science and Technology, Hong Kong, in 2001. He is a senior researcher in Livesforce Co., Ltd, Hangzhou, China. His research interests are 3D modeling, simulation and computer-aided design applied in toy and garment industry.

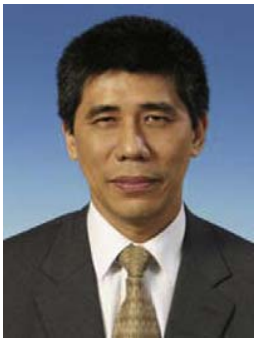

Ming-Fai Yuen received his Ph.D. in Mechanical Engineering from Bristol University, in 1977. He is a Professor in Hong Kong University of Science and Technology (HKUST). His research interests include CAD/CAM/CAE and Electronic Packaging. He had served as the Associate Dean of Engineering, the founding Director of the CAD/CAM Facility and the founding Director of the EPACK Laboratory at HKUST. Professor Yuen is currently holding two concurrent administrative positions at HKUST: Acting Vice-President of Research and Development, and President and CEO of HKUST R\&D Corporation. Professor Yuen is a Fellow of Institution of Mechanical Engineers, UK. 